\title{
UNIVERSITY OF ALBERTA
}

FACULTY OF ARTS

Department of Economics

Working Paper No. 2017-05

\section{Optimal Procurement of Distributed Energy Resources}

\author{
David P. Brown \\ University of Alberta
}

\author{
David E. M. Sappington \\ University of Florida
}

July 2017

Copyright to papers in this working paper series rests with the authors and their assignees. Papers may be downloaded for personal use. Downloading of papers for any other activity may not be done without the written consent of the authors.

Short excerpts of these working papers may be quoted without explicit permission provided that full credit is given to the source.

The Department of Economics, the Institute for Public Economics, and the University of Alberta accept no responsibility for the accuracy or point of view represented in this work in progress. 


\title{
Optimal Procurement of Distributed Energy Resources
}

by

\author{
David P. Brown* and David E. M. Sappington**
}

\begin{abstract}
We analyze the optimal design of policies to motivate electricity distribution companies to adopt efficient distributed energy resources (DER) and manage associated project costs. The optimal policy often entails a bias against new DER projects and implements considerable cost sharing when DER projects are undertaken in order to limit the utility's rent. Failure to adequately tailor the degree of cost sharing to the prevailing environment can raise procurement costs substantially. It can be optimal to reward a distribution company with more than the cost saving it achieves.
\end{abstract}

Keywords: distributed energy resources, procurement, regulation

JEL Codes: L51, L94

July 2017

* Department of Economics, University of Alberta, Edmonton, Alberta T6G 2H4 Canada (dpbrown@ualberta.ca).

** Department of Economics, University of Florida, Gainesville, FL 32611 USA USA (sapping@ufl.edu).

Support from the Government of Canada's Canada First Research Excellence Fund under the Future Energy Systems Research Initiative is gratefully acknowledged. 


\section{Introduction}

The role of electricity distribution companies has been changing in recent years and continues to evolve. Historically, these companies primarily arranged for the transport of electricity from a central generation site to remote customer locations. Today, electricity often is produced at sites other than the primary generation point, and distributed generation in the form of rooftop solar panels is expanding rapidly. Remote storage of electricity also exhibits ever-increasing potential to complement or substitute for distributed and centralized generation. In addition, improving ability to manage the demand for electricity can reduce the need for increased network transmission and distribution capacity (e.g., Ruester et al., 2014; Jenkins and Perez-Arriaga, 2017).

Advances in distributed generation, distributed storage, energy efficiency, and demand management offer the prospect of less costly and potentially more reliable electricity supply. ${ }^{1}$ To take full advantage of new opportunities as they emerge, regulators would like to motivate distribution utilities to first determine which new projects that entail distributed energy resources (DER) are superior to more traditional modes of operation, and then work diligently to integrate the most promising new DER projects into utility operations in a cost-effective manner. ${ }^{2}$ Such motivation can be difficult to provide, though, because regulators typically have limited knowledge of both the potential gains from new DER projects and the utility's ability to manage project costs (e.g., CPUC, 2016a). If regulators do not structure financial incentives appropriately, utilities may not implement the most promising projects and may not work diligently to manage the costs of projects that are implemented.

Regulators have adopted different policies to compensate utilities for the DER projects they pursue. Some regulators tend to follow the principles that underlie standard rate

\footnotetext{
${ }^{1}$ To illustrate, Consolidated Edison is spending approximately $\$ 200$ million on DERs in New York City to replace tradititonal network investment that would have cost nearly five times that amount (New York Public Service Commission (NYPSC), 2014b).

${ }^{2}$ California has adopted a pilot program that requires the state's major utilities to implement DER alternatives to traditional capital investment (California Public Utilities Commission (CPUC), 2016b). Proposed legislation in the Commonwealth of Massachussets (2017) would require utilities to consider "non-wire" alternatives before undertaking new investments in the electricity distribution grid
} 
of return regulation, sometimes permitting a relatively high return on DER projects to encourage their adoption despite the uncertainties they introduce (e.g., California Public Service Commission, 2016a). Other regulators have implemented policies that effectively require the utility and its customers to share unanticipated cost savings or cost overruns on DER projects (e.g., NYPSC, 2015, 2016).

The purpose of this research is to analyze the optimal design of regulatory policy in a stylized setting where the utility can choose between a core project and a non-core DER project. The core project can be viewed as a standard infrastructure investment that increases the utility's output. (The output can be viewed as the amount of electricity or the level of service quality that the utility delivers, for example.) The non-core DER project is an alternative, less standard means to achieve the same outcome. The cost of implementing the core project is known to all parties. In contrast, the utility has privileged information about the likely cost of the non-core project and about the efficacy of the utility's effort to manage project costs.

We characterize the regulatory policy that minimizes the expected cost of securing the required increase in output. We focus on how the details of the optimal compensation policy vary with the environment in which it is implemented. Relevant environmental features include the prevailing information asymmetry about likely costs under the non-core project and the efficacy of the utility's cost-containment effort. We find that the optimal policy often reduces expected procurement cost well below the level secured with standard costreimbursement policies. We also find that substantial reductions in procurement costs can be secured by tailoring the compensation structure to the environment in which it is implemented (e.g., by imposing less cost sharing for DER projects that are initiated by the utility rather than an unaffiliated third-party entity).

In some jurisdictions, regulators offer utilities a menu of optional compensation structures in an attempt to induce the utilities to truthfully reveal their investment needs. ${ }^{3}$ We compare

${ }^{3}$ See Crouch (2006), Joskow (2008), and Ofgem (2009), for example. 
optimal policies and industry outcomes when the regulator can, and cannot, offer such a menu of compensation structures. We find that although procurement costs often do not increase substantially when the regulator is unable to offer policy options, utility profit can increase dramatically. We also find that the regulator often will induce the utility to implement the core project even when its cost exceeds the expected cost of the non-core project. This bias toward the core project helps to limit the utility's incentive to understate the potential gains from the non-core project.

Our analysis also demonstrates the optimality of compensation structures that typically are not afforded serious consideration. Specifically, we identify conditions under which it is optimal to award the utility more than the full amount of any cost reduction it achieves. The large reward for cost reduction (and corresponding relatively limited compensation when a cost reduction is not achieved) can limit the utility's incentive to exaggerate innate costs and thereby exaggerate the efficacy of its cost-containment effort.

Our study is not the first to analyze the design of regulatory policy to promote efficient network improvements, potentially including DER projects. ${ }^{4}$ Costa et al. (2017), for example, document a utility's reluctance to undertake investments that do not expand its rate base in the presence of a generous allowed rate of return on the authorized rate base. We abstract from different rate-base treatments of investment in order to focus on the difficulties created by asymmetric information about likely project costs and the efficacy of the utility's cost-containment efforts.

Crouch (2006) analyzes the menus of optional cost-sharing plans that the UK electricity regulator, Ofgem, employs to induce utilities to reveal their investment needs (potentially including DER projects) and to control ensuing costs. Cossent and Gomez (2013) extend Crouch (2006)'s analysis of Ofgem's policy and demonstrate how the policy's principles might be employed to improve regulatory policy in the Spanish electricity distribution sector.

\footnotetext{
${ }^{4}$ Many studies (e.g., Couture and Gagnon, 2010; Yamamoto, 2012; Poullikkas, 2013; Brown and Sappington, 2017a) examine the impact of policies like net metering and feed-in tariffs on DER investment. Our distinct focus is on how to motivate utilities to integrate and manage DER projects efficiently.
} 
Jenkins and Perez-Arriaga (2017) augment Cossent and Gomez (2013)'s analysis with a simulated large-scale urban electricity distribution network that regulators can employ to better assess the utility's likely investment needs and production costs.

These studies focus on the properties of policies that induce the utility to truthfully reveal its investment needs while preserving incentives for cost reduction. We, too, analyze regulatory policies that induce the utility to reveal its superior knowledge of project potential while providing incentives for cost management. We also analyze the optimal design of such policies. We model formally both the prevailing information asymmetry and the utility's ability to reduce operating costs. ${ }^{5}$ Doing so allows us to determine how the optimal compensation policy varies with the environment in which it is implemented and with the instruments that are available to the regulator. ${ }^{6}$ We are also able to quantify the substantial potential gains from tailoring the regulatory policy to the environment in which it is implemented. ${ }^{7}$

The analysis proceeds as follows. Section 2 describes the key elements of the model. Section 3 identifies the optimal procurement policy in benchmark settings. Section 4 characterizes the optimal policy in the setting of primary interest when the regulator can afford the utility a choice among compensation structures. Section 5 provides the corresponding characterization when such a choice is not feasible, and discusses the implications for procurement costs. Section 6 reviews the policy implications of our analysis. Section 7 considers an extension of the basic model. Section 8 summarizes our findings, discusses extensions of our analysis, and suggests directions for additional research. The Appendix provides the

\footnotetext{
${ }^{5}$ Given the subtleties involved in characterizing optimal regulatory policy, we analyze a much more streamlined model of the relevant environment than the sophisticated resource planning model that Jenkins and Perez-Arriaga (2017) analyze.

${ }^{6}$ Jenkins and Perez-Arriaga (2017, p. 85) observe that under policies like the one Ofgem has implemented, "the regulator has significant flexibility and discretion to ... balance the fundamental regulatory tradeoffs between allocative efficiency (extracting rents from the utility) and productive efficiency (providing incentives for cost savings)." We build upon this observation by determining how the regulator optimally employs this flexibility and discretion to minimize expected procurement costs.

${ }^{7}$ Although we believe many of our findings are novel, the basic elements of our theoretical analysis are not new. Related models that include both ex ante information asymmetry and ex post unobserved costcontainment effort by the utility date back at least to Sappington (1982). Also see Laffont and Tirole (1993) and Laffont and Martimort (2002), for example.
} 
proofs of all formal conclusions in the text.

\section{The Model}

We consider a setting where the utility can secure a specified level of output by implementing either a traditional ("core") project or a non-traditional ("non-core") project. Output might constitute increased delivery of electricity or improved service quality, for instance. The core project might entail expanding the utility's distribution facilities to relieve local network congestion, for example. The non-core project might entail securing additional electricity from remote locations or initiating programs to reduce peak demand for electricity, for instance. Because the core project entails relatively familiar activities, the cost of the project $\left(c_{0}\right)$ is assumed to be known and deterministic. ${ }^{8}$ In contrast, the final cost of the non-core project is not known with certainty at the time it is undertaken.

For simplicity, the final cost of the non-core project is assumed to be either low $(\underline{c})$ or high $(\bar{c})$, where $\underline{c}<c_{0}<\bar{c}$. The probability that the realized cost is low, $p(e, \theta) \in(0,1)$, varies with the innate project potential, $\theta \in\left\{\theta_{1}, \theta_{2}\right\}$, and with the utility's cost-containment effort, $e \geq 0$. For any given level of $e \geq 0$, the low cost realization $(\underline{c})$ is more likely under the non-core project when it has high potential $\left(\theta_{2}\right)$ than when it has low potential $\left(\theta_{1}\right)$. Formally, $p\left(e, \theta_{2}\right)>p\left(e, \theta_{1}\right)$ for all $e \geq 0$. Furthermore, unless otherwise noted, the marginal impact of cost-containment effort is assumed to be more pronounced under the high-potential non-core project than under its low-potential counterpart, i.e., $p_{e}\left(e, \theta_{2}\right) \geq p_{e}\left(e, \theta_{1}\right)>0$ for all $e \geq 0 .{ }^{9}$ In addition, diminishing returns to cost-containment effort are less pronounced when $\theta=\theta_{2}$ than when $\theta=\theta_{1} \cdot{ }^{10}$ Thus, innate expected cost (i.e., expected cost in the absence of any cost-containment effort) is lower and the potential to contain costs is more pronounced under the non-core project when $\theta=\theta_{2}$ than when $\theta=\theta_{1}$.

\footnotetext{
${ }^{8}$ This cost includes the expenses associated with installing, maintaining, and operating efficiently relevant additions to the utility's infrastructure.

${ }^{9}$ Here and throughout the ensuing analysis, the subscript " $e$ " denotes the partial derivative with respect to $e$. Section 7 discusses the changes that can arise when $p_{e}\left(e, \theta_{2}\right)<p_{e}\left(e, \theta_{1}\right)$ for all $e \geq 0$.

${ }^{10}$ Formally, $p_{e e}\left(e, \theta_{i}\right) \leq 0$ for $i=1,2$ and $p_{e e}\left(e, \theta_{2}\right) \geq p_{e e}\left(e, \theta_{1}\right)$ for all $e \geq 0$, where the subscript "ee" denotes the second partial derivative with respect to $e$.
} 
The regulator and the utility both know how $e$ and $\theta$ affect the likelihood of securing the low cost realization under the non-core project (i.e., the functional form of $p(e, \theta)$ is common knowledge). However, only the utility observes the realization of $\theta$. The regulator knows only that $\theta=\theta_{i}$ with probability $\phi_{i} \in(0,1)$ for $i=1,2$, where $\phi_{1}+\phi_{2}=1$.

$D(e)$ is the personal cost the utility incurs when it delivers cost-containment effort $e$. $D(\cdot)$ includes the opportunity cost the utility's executives and managers incur when they devote their scarce time and energy to increasing the likelihood of the low cost realization under the non-core project. $D(\cdot)$ is an increasing, convex function of $e \cdot{ }^{11}$ The regulator, like the utility, knows the functional form of $D(\cdot)$. However, the regulator cannot observe the level of effort the utility supplies or the corresponding effort cost the utility incurs. Consequently, the regulator cannot elicit cost-containment effort by reimbursing the utility directly for the effort it supplies. Instead, the regulator must motivate the utility to deliver $e$ by promising the utility a higher level of profit when $\underline{c}$ is realized than when $\bar{c}$ is realized under the non-core project.

The utility's profit is the difference between: (i) the payment $(r)$ it receives from the regulator; and (ii) the sum of the realized production cost and any relevant effort cost. $r_{0}$ denotes the payment the regulator delivers to the utility when it implements the core project. Corresponding payments when the utility implements the non-core project can vary with the realized project cost. These payments also may vary with the particular compensation schedule the utility selects. We initially consider a setting where the regulator can design two distinct compensation schedules and assign a schedule to the utility based on its report of the non-core project's potential. Formally, when the utility reports $\theta=\theta_{j}$, it is instructed to undertake the non-core project with probability $\psi_{j} \in\{0,1\}$ and the core project with probability $1-\psi_{j}$. If it undertakes the non-core project after reporting $\theta=\theta_{j}$, the utility is paid $\underline{r}_{j}$ if the realized project cost is $\underline{c}$, and it is paid $\bar{r}_{j}$ if the realized cost is $\bar{c}$. Therefore, the utility's expected profit when $\theta=\theta_{i}$ and the utility reports $\theta=\theta_{j}$ is:

\footnotetext{
${ }^{11}$ Formally, $D(0)=0, D^{\prime}(e) \geq 0$, and $D^{\prime \prime}(e)>0$ for all $e \geq 0$, where the first inequality holds strictly for all $e>0$.
} 


$$
\begin{aligned}
\Pi_{j}\left(\theta_{i}\right) & =\psi_{j} \pi_{j}^{n}\left(\theta_{i}\right)+\left[1-\psi_{j}\right]\left[r_{0}-c_{0}\right], \text { where } \\
\pi_{j}^{n}\left(\theta_{i}\right) & \equiv \max _{e \geq 0}\left\{p\left(e, \theta_{i}\right)\left[\underline{r}_{j}-\underline{c}\right]+\left[1-p\left(e, \theta_{i}\right)\right]\left[\bar{r}_{j}-\bar{c}\right]-D(e)\right\} .
\end{aligned}
$$

The regulator seeks to minimize the expected cost of procuring the requisite level of output. The regulator's formal problem in this setting, $[\mathrm{RP}]$, is:

$$
\underset{r_{0}, \underline{r}_{i}, \bar{r}_{i}, \psi_{i} \in\{0,1\}}{\operatorname{Minimize}} \sum_{i=1}^{2} \phi_{i} \psi_{i}\left\{p\left(e_{i}, \theta_{i}\right) \underline{r}_{i}+\left[1-p\left(e_{i}, \theta_{i}\right)\right] \bar{r}_{i}\right\}+\left[1-\phi_{1} \psi_{1}-\phi_{2} \psi_{2}\right] r_{0}
$$

subject to, for $i, j \in\{1,2\}(j \neq i)$ :

$$
\Pi_{i}\left(\theta_{i}\right) \geq 0 \text { and } \Pi_{i}\left(\theta_{i}\right) \geq \Pi_{j}\left(\theta_{i}\right)
$$

$$
\text { where } e_{i} \equiv \underset{e \geq 0}{\arg \max }\left\{p\left(e, \theta_{i}\right)\left[\underline{r}_{i}-\underline{c}\right]+\left[1-p\left(e, \theta_{i}\right)\right]\left[\bar{r}_{i}-\bar{c}\right]-D(e)\right\}
$$

Expression (3) reflects the regulator's objective to minimize expected procurement cost. The first inequality in expression (4) ensures that the utility always secures at least its reservation level of expected profit, which is normalized to zero. The second inequality in expression (4) ensures that the utility will report the realization of $\theta$ truthfully. ${ }^{12}$

The interaction between the regulator and the utility proceeds as follows. First, the utility learns the realization of $\theta$. Second, the regulator specifies the procurement policy, which consists of payments to the utility $(r)$ and implementation probabilities $(\psi)$ that can vary with the utility's report of $\theta$. Third, the utility reports $\theta$ and undertakes the associated project. Fourth, the utility chooses its preferred level of cost-containment effort. ${ }^{13}$ Fifth, the project cost is realized and the regulator delivers the promised payment to the utility.

It will be helpful in the ensuing analysis to illustrate the magnitudes of selected qualitative effects. To do so, we introduce the following baseline setting, which is intended to serve as a simple representative example of the more general environment under consideration.

\footnotetext{
${ }^{12}$ The Revelation Principle (e.g., Myerson, 1979) ensures this formulation is without loss of generality. The value of $r_{0}$ could conceivably vary with the utility's report of $\theta$. However, it can be shown that such variation is never strictly valuable for the regulator in the present setting where $c_{0}$ is common knowledge.

${ }^{13}$ This effort is normalized to 0 under the core project. Alternatively, this effort can be taken to be $e_{0}>0$ and cost $c_{0}$ can be viewed as the sum of physical production cost and the utility's effort cost $D\left(e_{0}\right)$.
} 
$\underline{\text { Baseline Setting: }} p(e, \theta)=\beta \theta+\alpha\left[\frac{e}{1+e}\right], D(e)=\frac{\delta}{2} e^{2}, c_{0}=100,000, \underline{c}=50,000$,

$$
\bar{c}=150,000, \theta_{1}=0.25, \theta_{2}=0.50, \delta=50, \alpha=\beta=\phi_{1}=0.5 .
$$

\section{Benchmark Settings}

Before proceeding to characterize the solution to $[\mathrm{RP}]$, we consider the outcomes that would arise in four hypothetical benchmark settings. To do so, it is helpful to introduce the following definitions:

$$
\begin{aligned}
& e_{i}^{*} \equiv \underset{e \geq 0}{\arg \min }\left\{p\left(e, \theta_{i}\right) \underline{c}+\left[1-p\left(e, \theta_{i}\right)\right] \bar{c}+D(e)\right\} \text { for } i=1,2, \text { and } \\
& E c_{i}^{*} \equiv p\left(e_{i}^{*}, \theta_{i}\right) \underline{c}+\left[1-p\left(e_{i}^{*}, \theta_{i}\right)\right] \bar{c}+D\left(e_{i}^{*}\right) .
\end{aligned}
$$

In words, $e_{i}^{*}$ is the cost-minimizing ("efficient") effort supply under the non-core project when $\theta=\theta_{i}$, and $E c_{i}^{*}$ is the corresponding ("efficient") expected full cost (including effort cost) of the project. We assume $E c_{2}^{*}<c_{0}$, so the high-potential non-core project always offers the prospect of lower expected cost than the core project. ${ }^{14}$

In the hypothetical full-information setting, the regulator shares the utility's knowledge of $\theta$ from the outset of their relationship and can verify the level of cost-containment effort the utility supplies under the non-core project. In the presence of such symmetric information, the regulator will implement the full-information outcome, under which: (i) the utility undertakes the non-core project when $\theta=\theta_{i}$ if and only if $E c_{i}^{*} \leq c_{0}$; (ii) the utility delivers the efficient level of cost-containment effort when it implements the non-core project; and (iii) $r_{0}=c_{0}$ and $\pi_{1}^{n}\left(\theta_{1}\right)=\pi_{2}^{n}\left(\theta_{2}\right)=0$, so the utility secures no rent. ${ }^{15}$ In the setting with known project potential, the regulator and the utility both observe the realization of $\theta$ at the outset of their interaction, but the regulator never observes the utility's supply of costcontainment effort. In the setting with verifiable effort, the regulator can verify the utility's

\footnotetext{
${ }^{14}$ If this were not the case, the regulator would minimize expected procurement cost by setting $r_{0}=c_{0}$ and requiring the utility to always undertake the core project.

${ }^{15}$ Effort verification implies that the regulator can document conclusively the level of effort the utility has supplied, and so can write a legally enforceable contract that links the utility's payment $(r)$ to its effort supply $(e)$.
} 
supply of cost-containment effort, but only the utility observes the realization of $\theta$. In the setting with ex ante contracting, the regulator can specify the regulatory policy before the utility observes the realization of $\theta$. The regulator never observes the realization of $\theta$ or the utility's effort supply in this setting.

Lemma 1. The regulator can secure the full-information outcome in the setting with known project potential, in the setting with verifiable effort, and in the setting with ex ante contracting.

Lemma 1 reflects the following considerations. ${ }^{16}$ When the regulator observes the realization of $\theta_{i}$ in the setting with known project potential, she can instruct the utility to undertake the project that entails the lowest efficient expected full cost and set payments $r_{0}=c_{0}$ and $\underline{r}_{i}=\bar{r}_{i}=E c_{i}^{*}$ for $i=1,2$. The fixed payment $E c_{i}^{*}$ induces the efficient effort supply and eliminates the utility's rent under the non-core project when $\theta=\theta_{i}$. When the regulator can verify the utility's effort supply in the setting with verifiable effort, she can promise to reimburse the utility's observed full cost of production (including effort costs). This policy always leaves the utility with zero profit, so it is willing to undertake the project with the lowest efficient expected full cost and to deliver the efficient effort supply. When the regulator can commit to a policy when she shares the utility's imperfect knowledge of the prevailing environment (in the setting with ex ante contracting), she can offer the utility a fixed payment equal to its efficient expected full cost. This payment eliminates the utility's expected rent and induces the utility to implement the project with the lowest efficient expected full cost and to deliver the efficient effort supply whenever it implements the non-core project.

In practice, regulators attempt to limit relevant information asymmetries in part by requiring utilities to provide detailed information about their network configuration and

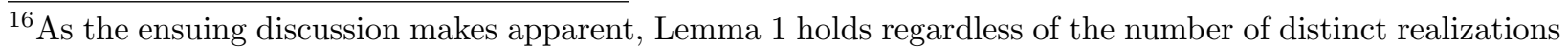
that $\theta$ can assume.
} 
location-specific capacities, ${ }^{17}$ thereby enabling engineering models and optimization software to simulate the benefits and costs of potential DER projects (MIT Energy Initiative, 2016; Jenkins and Perez-Arriaga, 2017). Lemma 1 implies that such efforts can be valuable. In practice, though, some information asymmetry is likely to persist, as is limited ability to measure accurately utility efforts to control the costs of non-core projects. Therefore, it is important to characterize the properties of optimal procurement policies when both of these frictions are present simultaneously.

\section{Findings When Optional Cost-Sharing Plans are Feasible.}

Conclusion 1 reports that the regulator can sometimes secure the full-information outcome even when she cannot observe $\theta$ or verify $e$.

Conclusion 1. Suppose $E c_{2}^{*}<c_{0}<E c_{1}^{*}$. Then $\psi_{1}=0, \psi_{2}=1, r_{0}=c_{0}$, and $\underline{r}_{2}=\bar{r}_{2}=E c_{2}^{*}$ at the solution to $[R P]$, so the regulator secures the full-information outcome.

Conclusion 1 considers a setting where the cost of the core project is less than the efficient expected full cost of the low-potential non-core project. In this setting, the regulator can induce the utility to always implement the project with the lowest efficient expected full cost by paying the utility: (i) $c_{0}$ if it undertakes the core project; and (ii) the smaller amount, $E c_{2}^{*}$, if it undertakes the non-core project. These payments ensure the non-core project is profitable for the utility if and only if $\theta=\theta_{2}$. Therefore, the utility will always undertake the project with the lowest efficient expected full cost and earn no rent. Furthermore, the fixed payment (as opposed to full cost reimbursement) that the utility faces when it undertakes the non-core project induces utility to deliver the cost-containment effort $\left(e_{2}^{*}\right)$ that minimizes expected full cost. ${ }^{18}$

\footnotetext{
${ }^{17}$ This is the case, for example, in California (CPUC, 2013), Hawaii (2014), and New York (NYPSC, 2014a, 2015, 2016).

${ }^{18}$ The regulator's ability to secure the full-information outcome in the setting of Conclusion 1 is an artifact of the simplifying assumption that $\theta$ is binary. When $\theta$ can assume more than two values and when $E c_{i}^{*}<c_{0}$ for at least two $\theta_{i}$ realizations, the regulator will face the constraining trade-off that we analyze next.
} 
The regulator faces a more challenging problem when $E c_{2}^{*}<E c_{1}^{*}<c_{0}$. In this setting, the regulator would have to set $\psi_{i}=1$ and $\underline{r}_{i}=\bar{r}_{i}=E c_{i}^{*}$ for $i=1,2$ to eliminate the utility's rent and always induce the efficient effort supply $e_{i}^{*}$. However, when afforded a choice between a relatively low fixed payment $\left(E c_{2}^{*}\right)$ and a higher fixed payment $\left(E c_{1}^{*}\right)$, the utility will always select the latter, and thereby secure rent when $\theta=\theta_{2}$.

$\pi_{1}^{n}\left(\theta_{2}\right)-\pi_{1}^{n}\left(\theta_{1}\right)$ is the incremental profit that accrues to the utility when $\theta$ is $\theta_{2}$ rather than $\theta_{1}$ under payment structure $\left(\underline{r}_{1}, \bar{r}_{1}\right)$. As Lemma 2 reports, this incremental profit increases as $\Delta_{1} \equiv \underline{r}_{1}-\underline{c}-\left(\bar{r}_{1}-\bar{c}\right)$ increases. The larger the incremental profit when $\underline{c}$ rather than $\bar{c}$ is realized, the larger the incremental profit the utility secures as the potential of the non-core project increases (due to the associated increase in the likelihood of realizing $\underline{c}$ for any given level of $e$ ). Lemma 2 refers to $e_{12} \equiv \underset{e \geq 0}{\arg \max }\left\{p\left(e, \theta_{2}\right)\left[\underline{r}_{1}-\underline{c}\right]+\left[1-p\left(e, \theta_{2}\right)\right]\left[\bar{r}_{1}-\bar{c}\right]-\right.$ $D(e)\}$.

Lemma 2. $\pi_{1}^{n}\left(\theta_{2}\right)>\pi_{1}^{n}\left(\theta_{1}\right)$ and $\frac{\partial}{\partial \Delta_{1}}\left\{\pi_{1}^{n}\left(\theta_{2}\right)-\pi_{1}^{n}\left(\theta_{1}\right)\right\}=p\left(e_{12}, \theta_{2}\right)-p\left(e_{1}, \theta_{1}\right)>0$ whenever $\Delta_{1}>0$.

Lemma 2 identifies the critical trade-off the regulator faces when $E c_{2}^{*}<E c_{1}^{*}<c_{0}$. Reducing the extent to which the utility shares realized costs with its customers by increasing $\Delta_{1}$ toward $\bar{c}-\underline{c}$ increases the utility's cost-containment effort toward $e_{1}^{*}$ and thereby reduces expected procurement cost when $\theta=\theta_{1} \cdot{ }^{19}$ However, the diminished cost sharing increases the profit the utility can secure under the $\left(\underline{r}_{1}, \bar{r}_{1}\right)$ payment structure when $\theta=\theta_{2}$, and thereby increases the payments $\left(\underline{r}_{2}\right.$ and/or $\left.\bar{r}_{2}\right)$ the regulator must promise to the utility to induce it to select the $\left(\underline{r}_{2}, \bar{r}_{2}\right)$ payment structure under the non-core project when $\theta=\theta_{2}$. As Conclusion 2 reports, the regulator optimally resolves this trade-off by implementing some cost sharing when $\theta=\theta_{1}$, i.e., by setting $\underline{r}_{1}$ below $\bar{r}_{1}$, so $\Delta_{1}<\bar{c}-\underline{c}$. Doing so increases procurement cost when $\theta=\theta_{1}$ by reducing $e_{1}$ below $e_{1}^{*}$, but reduces procurement cost by a

\footnotetext{
${ }^{19}$ When $\underline{r}_{1}<\bar{r}_{1}$ and so $\Delta_{1}<\bar{c}-\underline{c}$, the utility shares costs with its customers in the sense that the customers effectively bear a fraction of the higher cost that arises when $\bar{c}$ rather than $\underline{c}$ is realized under the non-core project.
} 
greater amount when $\theta=\theta_{2}$ by diminishing the rent that must be afforded the utility when $\theta=\theta_{2}$.

Conclusion 2. If $\psi_{1}=\psi_{2}=1$ at the solution to [RP], then $\bar{r}_{1}>\underline{r}_{1}\left(\right.$ so $\left.\Delta_{1} \in(0, \bar{c}-\underline{c})\right)$, $\pi_{1}^{n}\left(\theta_{1}\right)=0, \bar{r}_{2}=\underline{r}_{2}$, and $\pi_{2}^{n}\left(\theta_{2}\right)=\pi_{1}^{n}\left(\theta_{2}\right)>0$.

Conclusion 2 reports that when the regulator always induces the utility to undertake the non-core project (so $\psi_{1}=\psi_{2}=1$ at the solution to [RP]), she does so by offering the utility a choice between a cost-sharing plan (with $\left.\underline{r}_{1}<\bar{r}_{1}\right)$ and a fixed payment $\left(\underline{r}_{2}=\bar{r}_{2}\right)$. The fixed payment, which induces the utility to deliver the efficient effort supply $\left(e_{2}^{*}\right)$ when $\theta=\theta_{2}$, is the smallest payment required to convince the utility not to adopt the cost-sharing contract when $\theta=\theta_{2}\left(\right.$ so $\left.\pi_{2}^{n}\left(\theta_{2}\right)=\pi_{1}^{n}\left(\theta_{2}\right)>0\right)$.

Lemma 3 reports that the regulator will always induce the utility to undertake the noncore project when the potential cost saving from implementing the non-core project when $\theta=\theta_{1}$ rather than the core project is sufficiently pronounced.

Lemma 3. At the solution to $[R P]:$ (i) $\psi_{2}=1$; and (ii) $\psi_{1}=1$ if $E c_{1}^{*}<\phi_{1} c_{0}+\phi_{2} E c_{2}^{*}$.

Corollary 1. $\psi_{1}=0$ at the solution to $[R P]$ if $c_{0}-E c_{1}^{*}$ is negative or if $c_{0}-E c_{1}^{*}$ is positive and sufficiently small.

Corollary 1 implies that the regulator may optimally induce the utility to undertake the core project when $\theta=\theta_{1}$ even when the efficient expected full cost under the low-potential $\left(\theta_{1}\right)$ non-core project is substantially less than $c_{0}$. The regulator will do so, for instance in the baseline setting except that $\phi_{1}=0.10$. In this setting, $E c_{1}^{*}=94,516<100,000=c_{0}$, but the regulator will induce the utility undertake the core project when $\theta=\theta_{1}$. Although doing so increases expected procurement cost above $E c_{1}^{*}$ in the (relatively unlikely) event that $\theta=\theta_{1}$, it reduces overall expected procurement costs by eliminating the utility's rent when $\theta=\theta_{2}$ (by setting $\left.\underline{r}_{2}=\bar{r}_{2}=E c_{2}^{*}\right)^{20}$

\footnotetext{
${ }^{20}$ The regulator will induce the utility undertake the core project when $\theta=\theta_{1}$ even when $E c_{1}^{*}$ is substantially
} 
When the regulator always induces the utility to undertake the non-core project, the incremental profit she awards the utility for realizing $\operatorname{cost} \underline{c}$ when $\theta=\theta_{1}$ (i.e., the value of $\left.\Delta_{1}\right)$ varies with the prevailing environment. Conclusion 3 reports that the regulator will increase $\Delta_{1}$ as she becomes more certain that $\theta=\theta_{1}$. The increase in $\Delta_{1}$ toward $\bar{c}-\underline{c}$ increases $e_{1}$ toward $e_{1}^{*}$ and thereby reduces expected procurement cost when $\theta=\theta_{1}$. The increase in $\Delta_{1}$ also increases procurement cost when $\theta=\theta_{2}$ by increasing the fixed payment $\left(\underline{r}_{2}=\bar{r}_{2}\right)$ that must be awarded the utility to induce it not to select the $\left(\underline{r}_{1}, \bar{r}_{1}\right)$ payment structure. However, the expected loss from the increased procurement cost when $\theta=\theta_{2}$ declines as $\phi_{2}$ declines. Conclusion 3, and Conclusions $4-7$ below, hold when $\left|D^{\prime \prime \prime}(e)\right|$ and $\left|p_{\text {eee }}\left(e, \theta_{i}\right)\right|$ are sufficiently small for all $e \geq 0$, for $i=1,2 .{ }^{21}$

Conclusion 3. $\frac{d \Delta_{1}}{d \phi_{1}}>0$ at the solution to $[R P]$ when $\psi_{1}=1$.

Conclusion 4 identifies conditions under which the regulator will reduce $\Delta_{1}$ as $\theta_{2}$ increases and $\theta_{1}$ declines, so the prevailing information asymmetry $\left(\theta_{2}-\theta_{1}\right)$ becomes more pronounced.

Conclusion 4. When $\psi_{1}=1$ at the solution to [RP]: (i) $\frac{d \Delta_{1}}{d \theta_{2}}<0$; and (ii) $\frac{d \Delta_{1}}{d \theta_{1}}>0$ so $\frac{d \Delta_{1}}{d\left(\theta_{2}-\theta_{1}\right)}<0$, if $\phi_{2}$ is sufficiently large or $\left|p_{e e}\left(e, \theta_{1}\right)\right|$ is sufficiently small for all $e \geq 0$.

An increase in $\theta_{2}$ (which increases $\theta_{2}-\theta_{1}$ ) increases the rate at which the utility's incremental rent when $\theta$ is $\theta_{2}$ rather than $\theta_{1}$ (i.e., $\left.\pi_{1}^{n}\left(\theta_{2}\right)-\pi_{1}^{n}\left(\theta_{1}\right)\right)$ increases with $\Delta_{1}$. Therefore, as $\theta_{2}$ increases, the regulator reduces $\Delta_{1}$ in order to reduce expected procurement cost when $\theta=\theta_{2}$

An increase in $\theta_{1}$ (which reduces $\theta_{2}-\theta_{1}$ ) reduces the rate at which an increase in $\Delta_{1}$ increases $\pi_{1}^{n}\left(\theta_{2}\right)-\pi_{1}^{n}\left(\theta_{1}\right)$. The corresponding reduced concern with the utility's rent when $\theta=\theta_{2}$ alone would lead the regulator to increase $\Delta_{1}$. If the increase in $\theta_{1}$ also increases the

further below $c_{0}$. She will do so, for instance in the baseline setting except that $\underline{c}=30,000, \bar{c}=170,000$, $\alpha=0.65, \delta=10$, and $\phi_{1}=0.10$. In this setting, $E c_{1}^{*}=67,833<100,000=c_{0}$.

${ }^{21}$ These limits on the magnitudes of relevant third derivatives help to ensure that the regulator's objective in $[\mathrm{RP}]$ is a concave function of $\Delta_{1}$. These limits constitute sufficient conditions for the Conclusions to hold, but generally are not necessary conditions (as the proofs of the Conclusions reveal). 
rate at which an increase in $\Delta_{1}$ increases $e_{1}$ toward $e_{1}^{*}$, then the regulator will increase $\Delta_{1}$. However, an increase in $\theta_{1}$ can reduce the rate at which an increase in $\Delta_{1}$ increases $e_{1}$ toward $e_{1}^{*}$ if $\left|p_{e e}\left(e, \theta_{1}\right)\right|$ is large. If this effect is sufficiently pronounced and if $\theta_{1}$ is sufficiently likely, the regulator could conceivably reduce $\Delta_{1}$ as $\theta_{1}$ increases. Conclusion 4 identifies conditions under which these latter considerations are outweighed by the regulator's reduced concern with the incremental rent that an increase in $\Delta_{1}$ generates as $\theta_{1}$ increases.

In essence, Conclusion 4 reports that as the relevant information asymmetry becomes more pronounced, the regulator often will implement more cost sharing (i.e., reduce $\Delta_{1}$ ) when $\theta=\theta_{1}$ to limit the utility's rent (and thus procurement cost) when $\theta=\theta_{2} \cdot{ }^{22}$

Conclusion 5 reports that the regulator will also reduce the incremental payment it delivers to the utility when $\underline{c}$ rather than $\bar{c}$ is realized as the maximum potential cost reduction under the non-core project $(\bar{c}-\underline{c})$ increases. An increase in $\bar{c}-\underline{c}$ increases the rent the utility derives from the $\left(\underline{r}_{1}, \bar{r}_{1}\right)$ payment structure, and thereby increases expected procurement cost when $\theta=\theta_{2}$. The regulator reduces this rent and the corresponding procurement cost by reducing $\underline{r}_{1}-\bar{r}_{1}$, so $\Delta_{1}$ increases by less than $\bar{c}-\underline{c}$ increases.

Conclusion 5. $\quad \frac{d\left(\underline{r}_{1}-\bar{r}_{1}\right)}{d(\bar{c}-\underline{c})}<0$ and $\frac{d \Delta_{1}}{d(\bar{c}-\underline{c})}<1$ when $\psi_{1}=1$ at the solution to [RP].

To understand how changes in the utility's cost of delivering cost-containment effort affect the optimal procurement policy, it is helpful to consider the hypothetical setting where the utility's effort cost $D(e)$ can vary with $\theta$. Specifically, suppose $D\left(e, \delta_{i}\right)$ is the utility's cost of delivering effort $e$ when $\theta=\theta_{i}$. Assume $\frac{\partial D\left(e, \delta_{i}\right)}{\partial \delta_{i}}>0$ and $\frac{\partial^{2} D_{e}\left(e, \delta_{i}\right)}{\partial e \partial \delta_{i}}>0$, so an increase in $\delta_{i}$ corresponds to an increase in the total and marginal cost of delivering $e$ when $\theta=\theta_{i}$, which induces the utility to reduce $e_{i}$, ceteris paribus.

Conclusion 6. When $\psi_{1}=1$ at the solution to $[R P]$ : (i) $\frac{d \Delta_{1}}{d \delta_{2}}>0$; and (ii) $\frac{d \Delta_{1}}{d \delta_{1}}>0$ when $\phi_{1}$ is sufficiently large, whereas $\frac{d \Delta_{1}}{d \delta_{1}}<0$ when $\phi_{2}$ is sufficiently large. Therefore, $\frac{d \Delta_{1}}{d \delta}>0$

\footnotetext{
${ }^{22}$ The reduction in $\Delta_{1}$ that arises as $\underline{r}_{1}-\bar{r}_{1}>0$ is reduced constitutes more pronounced cost sharing in the sense that consumers effectively bear a larger fraction of the higher cost that arises when $\bar{c}$ rather than $\underline{c}$ is realized.
} 
when $\phi_{1}$ is sufficiently large, where $\delta=\delta_{1}=\delta_{2}$.

Conclusion 6 reports that as cost-containment effort becomes more onerous for the utility to supply when $\theta=\theta_{2}$, the utility secures less rent from the $\left(\underline{r}_{1}, \bar{r}_{1}\right)$ cost-sharing contract when $\theta=\theta_{2}$. The regulator's corresponding reduced concern with the utility's rent when $\theta=\theta_{2}$ leads her to increase $\Delta_{1}$ toward $\bar{c}-\underline{c}$ in order to reduce expected procurement cost when $\theta=\theta_{1}$ by inducing the utility to increase $e_{1}$ toward $e_{1}^{*}$.

As cost-reducing effort becomes more onerous for the utility to supply when $\theta=\theta_{1}$, the utility secures more rent from the $\left(\underline{r}_{1}, \bar{r}_{1}\right)$ cost-sharing contract when $\theta=\theta_{2}$. The regulator's corresponding increased concern with the utility's rent when $\theta=\theta_{2}$ leads her to reduce $\Delta_{1}$, particularly when $\phi_{2}$ is relatively large. However, an increase in $\delta_{1}$ leads the utility to reduce $e_{1}$. To ensure $e_{1}$ does not decline unduly, the regulator might increase $\Delta_{1}$, particularly when $\phi_{1}$ is relatively large. Together, these considerations imply that a systematic increase in effort costs often will increase $\Delta_{1}$, particularly when $\phi_{1}$ is large. ${ }^{23}$

The cost sharing that the regulator optimally implements when $\theta=\theta_{1}$ also varies with the efficacy of the utility's cost-containment effort. Conclusion 7 characterizes the relationship between $\Delta_{1}$ and effort efficacy $(\alpha)$ in the special case where $\alpha$ does not vary with $e$ or $\theta$.

Conclusion 7. If $p_{e}\left(e, \theta_{1}\right)=p_{e}\left(e, \theta_{2}\right)=\alpha>0$ for all $e \geq 0$, then $\frac{d \Delta_{1}}{d \alpha}>0$ when $\psi_{1}=1$ at the solution to $[R P]$.

Conclusion 7 reflects the fact that as $\alpha$ increases, effort becomes more effective at securing cost $\underline{c}$ under the non-core project. Consequently, the regulator induces more effort by reducing the extent of cost sharing (i.e., by increasing $\Delta_{1}$ ).

Table 1 illustrates the magnitudes of the qualitative effects identified in Conclusions 3 7. The first column in the table identifies the variable of interest. $E\{\pi\}$ denotes the utility's expected profit. $E\{r\}$ denotes expected procurement cost, i.e., expected payment to the

$\overline{23 \frac{d \Delta_{1}}{d \delta} \text { can be strictly positive even when } \phi_{1}}$ is relatively small. This is the case, for example, in the baseline setting with the exception that $\underline{c}=30,000, \bar{c}=130,000$, and $\phi_{1}=0.25$. 
utility. (Recall that procurement cost is $c_{0}=100,000$ when the utility undertakes the core project.) The second column records the value of the variable at the solution to $[\mathrm{RP}]$ in the baseline setting. Columns $3-7$ report how this value changes as $\phi_{1}, \theta_{2}, \bar{c}, \delta$, and $\alpha$ increase by $10 \%$ above their values in the baseline setting (holding all other parameters at their values in the baseline setting). ${ }^{24}$

\begin{tabular}{|c|c|c|c|c|c|c|}
\hline & \multirow{2}{*}{$\begin{array}{l}\text { Baseline } \\
\text { Setting }\end{array}$} & \multicolumn{5}{|c|}{$10 \%$ Increase in } \\
\hline & & $\phi_{1}$ & $\theta_{2}$ & $\bar{c}$ & $\delta$ & $\alpha$ \\
\hline$\underline{r}_{1}$ & 58,625 & 59,736 & 57,614 & & 58,735 & 58,255 \\
\hline $\bar{r}_{1}$ & 141,171 & 139,853 & 142,353 & & 141,170 & 139,936 \\
\hline$\Delta_{1}$ & 17,454 & 19,883 & 15,261 & & 17,565 & 18,319 \\
\hline$\underline{r}_{2}=\bar{r}_{2}$ & 84,199 & 84,502 & 81,806 & 86,475 & 84,423 & 79,782 \\
\hline$E\{\pi\}$ & 1,091 & 1,119 & 1,145 & 0 & 1,098 & 1,145 \\
\hline$E\{r\}$ & 90,362 & 90,964 & 89,316 & 93,238 & 90,601 & 85,905 \\
\hline
\end{tabular}

Table 1. Effects of Parameter Changes in the Baseline Setting.

\section{Findings When Optional Cost-Sharing Plans are Not Feasible.}

In practice, it is relatively uncommon for regulators to offer utilities an explicit choice among payment structures. ${ }^{25}$ Consequently, it is important to determine how the optimal procurement policy changes when the regulator can only specify a single payment structure for the utility when it implements the non-core policy. Let [RP1] denote the regulator's problem in this setting. ${ }^{26}$ Also in this setting, let: (i) $\underline{r}$ and $\bar{r}$, respectively, denote the pay-

${ }^{24}$ The entries in Table 1 and in all subsequent tables are rounded to the nearest whole number. The blank entries in the fifth column of Table 1 reflect the fact that when $\bar{c}$ increases by $10 \%$ in the baseline setting, the regulator induces the utility to undertake the core project when $\theta=\theta_{1}$. Brown and Sappington (2017b) provide additional characterization of the optimal procurement policy as model parameters change.

${ }^{25}$ The U.S. Federal Communications Commission afforded suppliers of telecommunications services a choice among payment structures in the 1990s (Sappington and Weisman, 1996, pp. 162-165). Ofgem has been offering electricity distribution companies a choice among compensation arrangements for capital expenditures since 2005 (Crouch, 2006).

${ }^{26}[\mathrm{RP} 1]$ is $[\mathrm{RP}]$ with the additional requirement that $\underline{r}_{1}=\underline{r}_{2}$ and $\bar{r}_{1}=\bar{r}_{2}$. 
ments delivered to the utility when $\operatorname{costs} \underline{c}$ and $\bar{c}$ are realized under the non-core project; (ii) $\pi^{n}\left(\theta_{i}\right) \equiv \max _{e \geq 0}\left\{p\left(e, \theta_{i}\right)[\underline{r}-\underline{c}]+\left[1-p\left(e, \theta_{i}\right)\right][\bar{r}-\bar{c}]-D(e)\right\}$ denote the utility's expected profit under the non-core project when $\theta=\theta_{i}$; and (iii) $\Delta \equiv \underline{r}-\underline{c}-(\bar{r}-\bar{c})$ denote the incremental profit the utility receives when cost $\underline{c}$, rather than cost $\bar{c}$, is realized under the non-core project.

Conclusion 8 identifies the optimal procurement policy in this setting when $E c_{1}^{*}$ is sufficiently large that the regulator only induces the utility to undertake the non-core project when the project has high potential $\left(\theta_{2}\right)$. In this case, the regulator offers the utility a choice between the core project with payment $r_{0}=c_{0}$ and the non-core project with fixed payment $E c_{2}^{*}$. This fixed payment (as opposed to cost reimbursement) induces the utility to supply the efficient level of cost-containment effort $\left(e_{2}^{*}\right)$ and eliminates the utility's rent when $\theta=\theta_{2}$. Because $E c_{2}^{*}<E c_{1}^{*}$, the fixed payment renders the low-potential non-core project unprofitable for the utility, so the utility implements the core project when $\theta=\theta_{1}$.

Conclusion 8. Suppose $c_{0}-E c_{1}^{*}$ is negative or $c_{0}-E c_{1}^{*}$ is positive and sufficiently small. Then $r_{0}=c_{0}$ and $\bar{r}=\underline{r}=E c_{2}^{*}\left(\right.$ so $\pi^{n}\left(\theta_{2}\right)=0$ ) at the solution to [RP1]. ${ }^{27}$

Conclusion 9 reports that when $E c_{1}^{*}$ is sufficiently far below $c_{0}$, the regulator always induces the utility to implement the non-core project. ${ }^{28}$ She does so with a cost sharing plan $(\Delta \in(0, \bar{c}-\underline{c}))$ that eliminates the utility's rent if and only if $\theta=\theta_{1}$. In contrast to the outcome when the regulator can introduce more than one compensation policy under the non-core project, this single cost sharing plan induces the utility to deliver less than the efficient level of cost-containment effort both when $\theta=\theta_{1}$ and when $\theta=\theta_{2}$.

\footnotetext{
${ }^{27} \mathrm{As}$ in the setting where the regulator can implement two distinct payment structures under the non-core project, the regulator may optimally induce the utility to implement the core project when $\theta=\theta_{1}$ even when $E c_{1}^{*}$ is substantially below $c_{0}$. This will be the case, for example in the setting identified immediately following the statement of Corollary 1.

${ }^{28}$ The remainder of the discussion in this section considers the setting where the regulator always induces the utility to implement the non-core project.
} 
Conclusion 9. If $c_{0}-E c_{1}^{*}$ is sufficiently large, the regulator will induce the utility to always implement the non-core project at the solution to [RP1] with a cost-sharing contract in which $\bar{r}>\underline{r}$, where $\pi^{n}\left(\theta_{2}\right)>\pi^{n}\left(\theta_{1}\right)=0$.

The cost sharing identified in Conclusion 9 reduces expected procurement cost relative to the fixed payment $\underline{r}=\bar{r}=E c_{1}^{*}$. This is the case because for a given level of costcontainment effort, the low cost realization $\underline{c}$ is more likely to arise when the non-core project has high potential $\left(\theta_{2}\right)$ than when it has low potential $\left(\theta_{1}\right)$. Consequently, by reducing $\underline{r}$ below $\bar{r}$, the regulator effectively secures for consumers a portion of the reduction in expected cost that arises when the non-core project has high potential $\left(\theta_{2}\right)$ rather than low potential $\left(\theta_{1}\right)$. At least for small levels of cost sharing, the corresponding reduction in expected procurement cost when $\theta=\theta_{2}$ outweighs the increase in expected procurement cost that arises when the cost sharing induces the utility to reduce $e_{i}$ below $e_{i}^{*}$ for $i=1,2 .{ }^{29}$

As Conclusion 10 reports, the regulator optimally implements less cost sharing when she can only offer a single compensation structure under the non-core project. This is the case because cost sharing in this instance reduces the utility's cost-containment effort both when $\theta=\theta_{2}$ and when $\theta=\theta_{1}$. In contrast, when the regulator can offer the utility a choice between compensation structures under the non-core project, the optimal choice includes a fixed payment $\left(\underline{r}_{2}=\bar{r}_{2}\right)$ that is just sufficient to induce the utility not to choose the cost-sharing plan (with $\underline{r}_{1}<\bar{r}_{1}$ ) when $\theta=\theta_{2}$. Consequently, although the cost sharing plan reduces $e_{1}$ below $e_{1}^{*}$, it does not reduce $e_{2}$ below $e_{2}^{*}$ as it does when the regulator only offers a single compensation structure under the non-core project. Conclusion 10 refers to $\Delta^{*}$ and $\Delta_{1}^{*}$, which are the values of $\Delta \equiv \underline{r}-\underline{c}-(\bar{r}-\bar{c})$ at the solution to [RP1] and $\Delta_{1} \equiv \underline{r}_{1}-\underline{c}-\left(\bar{r}_{1}-\bar{c}\right)$ at the solution to $[\mathrm{RP}]$, respectively.

Conclusion 10. $\Delta^{*}>\Delta_{1}^{*}$.

The magnitude of $\Delta^{*}-\Delta_{1}^{*}$ can be viewed as the reduction in the extent of cost sharing ${ }^{29}$ Observe that $\frac{d}{d \Delta}\left\{p\left(e_{1}, \theta_{1}\right) \underline{c}+\left[1-p\left(e_{1}, \theta_{1}\right)\right] \bar{c}+D(e)\right\}_{\Delta=\bar{c}-\underline{c}}=\left[-p_{e}\left(e_{1}^{*}, \theta_{1}\right)(\bar{c}-\underline{c})+D^{\prime}\left(e_{1}^{*}\right)\right] \frac{d e_{1}^{*}}{d \Delta}=$ 0 . 
that is optimally implemented when $\theta=\theta_{1}$ if the regulator can only offer a single compensation structure under the non-core project. The magnitude of the reduced cost sharing can be substantial under plausible conditions. As Table 2 reports, $\Delta^{*}$ is more than four times larger than $\Delta_{1}^{*}$ in the baseline setting. ${ }^{30}$ The large increase in $\Delta^{*}$ above $\Delta_{1}^{*}$ provides substantial rent to the utility when $\theta=\theta_{2}$, causing the utility's expected profit $(E\{\pi\})$ to increase more than four-fold. However, because substantially more cost-containment effort is induced, ${ }^{31}$ expected procurement cost $(E\{r\})$ increases by only $2.64 \%$ in this setting. ${ }^{32}$

\begin{tabular}{|c|c|c|}
\hline & $\begin{array}{c}\text { Two Non-Core } \\
\text { Contracts Feasible }\end{array}$ & $\begin{array}{c}\text { One Non-Core } \\
\text { Contract Feasible }\end{array}$ \\
\cline { 2 - 3 } & $\Delta_{1}^{*}=17,454$ & $\Delta^{*}=70,265$ \\
\hline$E\{\pi\}$ & 1,091 & 4,392 \\
\hline$E\{r\}$ & 90,362 & 92,751 \\
\hline
\end{tabular}

Table 2. Outcomes in the Baseline Setting.

The key forces that influence the optimal magnitude of $\Delta_{1}$ when optional cost-sharing plans are feasible continue to influence the optimal magnitude of $\Delta$ when optional plans are not feasible. One additional effect also arises in this latter setting. Now, in addition to influencing the utility's cost containment effort when $\theta=\theta_{1}$ and the utility's rent when $\theta=\theta_{2}$, the value of $\Delta$ affects the utility's cost containment effort when $\theta=\theta_{2}$. This additional role for $\Delta$ could conceivably alter some of the qualitative conclusions reported in Conclusions $3-7$. It generally does not do so under plausible conditions, though.

\footnotetext{
${ }^{30}$ In the baseline setting: (i) $\underline{r}_{1}=58,625$ and $\bar{r}_{1}=141,171$ at the solution to [RP]; and (ii) $\underline{r}=81,849$ and $\bar{r}=111,585$ at the solution to [RP1].

${ }^{31}$ In the baseline setting: (i) $e_{1}=8.24$ at the solution to [RP1]; and (ii) $e_{1}=4.94$ at the solution to [RP].

${ }^{32}$ The qualitative conclusions reflected in Table 2 are quite robust to variation in model parameters. (See Brown and Sappington (2017b) for details.) The finding that a contract designer (here, the regulator) does not experience a major reduction in expected welfare when she is limited to offering a single compensation structure in the presence of adverse selection (here, incomplete information about $\theta$ ) is consistent with the findings of Reichelstein (1992), Bower (1993), and Rogerson (2003). Chu and Sappington (2007) identify a setting in which the expected welfare loss can be pronounced, depending on the distribution of the relevant environmental parameter (here, $\theta$ ).
} 
To illustrate, observe that as $\phi_{2}$ increases, the regulator might conceivably increase $\Delta$ to induce the utility to increase $e_{2}$ toward $e_{2}^{*}$ when $\theta=\theta_{2}$. However, the regulator often will reduce $\Delta$ instead in order to reduce the utility's rent when $\theta=\theta_{2}$, just as in the setting of Conclusion 3. This will be the case, for instance, when the impact of increased effort on the probability of realizing the low $\operatorname{cost} \underline{c}$ under the non-core project is not much larger when $\theta=\theta_{2}$ than when $\theta=\theta_{1}$. Conclusion 13 in the Appendix provides the formal details. The Appendix also reports that the direct counterparts to Conclusions $5-7$ persist in the present environment, and identifies the additional structure (primarily limits on the magnitudes of relevant second and third derivatives) that is sufficient to ensure the counterpart to Conclusion 4 holds in the present setting.

\section{Policy Implications.}

Three policy implications of the foregoing findings merit emphasis. First, cost sharing can be an important component of an optimal procurement policy. In practice, utility compensation for DER investments often reflects standard rate of return principles. ${ }^{33}$ As is well known, guaranteed rates of return provide limited incentive to control costs. Our analysis reveals that the utility may command rent from its superior knowledge of the likely costs of DER projects, but the rent is optimally afforded in a manner that provides incentives for cost control (including payments that do not vary with realized costs). ${ }^{34}$ Thus, financial rewards for realizing low costs under non-core projects, like the rewards offered in New York State, can have considerable merit (NYPSC, 2014a, 2015, 2016).

To illustrate the potential gains from implementing optimally designed cost-sharing contracts, consider the baseline setting. If the regulator fully reimbursed the utility for realized production costs in this setting (so $\underline{r}_{1}=\underline{r}_{2}=50,000$ and $\bar{r}_{1}=\bar{r}_{2}=150,000$ ), the utility would deliver no cost-containment effort and expected procurement costs would be 131,250

\footnotetext{
${ }^{33}$ In California, for instance, utilities typically are awarded a return in excess of the normal allowed rate of return for undertaking DER projects (CPUC, 2016b).

${ }^{34}$ Fixed payments generally will not be optimal if the utility is averse to risk. The impact of risk aversion (for both the utility and the regulator) on the optimal procurement policy merits further study.
} 
if the utility always implemented the non-core project. Under the optimal procurement policy in this setting (i.e., at the solution to $[\mathrm{RP}]$, where $\underline{r}_{1}=58,625, \bar{r}_{1}=141$, 171 , and $\left.\underline{r}_{2}=\bar{r}_{2}=84,199\right)$, expected procurement cost would be 90,362. Thus, implementation of a cost-reimbursement policy rather than the optimal cost-sharing policy could cause expected procurement costs to increase by $45.2 \%$ in this setting. ${ }^{35}$

Second, utility earnings (and to a lesser extent procurement costs) can vary with the regulator's ability to offer the utility a choice among compensation structures under the non-core project. As Table 2 illustrates, the utility may secure a substantial increase in profit when the regulator is only able to offer a single compensation structure. The increased profit arises when the regulator offers a relatively large incremental reward for realizing $\underline{c}$ (i.e., relatively limited cost sharing) in order to induce the utility to deliver a substantial amount of cost-containment effort under the low-potential $\left(\theta_{1}\right)$ non-core project. The relatively large level of induced effort serves to reduce expected cost under the non-core project when $\theta=\theta_{1}$, which often prevents a substantial expected increase in procurement cost. Thus, while procurement costs may not rise dramatically when the regulator is unable to tailor the compensation structure to the prevailing potential of the non-core project, the utility may experience a pronounced increase in expected profit. This finding may help to explain why regulators seldom offer utilities an explicit choice among compensation structures in practice. $^{36}$

Third, the amount of cost sharing that is optimally induced varies with many elements of the prevailing environment, including, for instance, whether the non-core DER project is internal (i.e., owned and operated by the utility) or external (i.e., secured from an entity other than the utility). ${ }^{37}$ In some jurisdictions, e.g., New York State, utilities are now generally

\footnotetext{
${ }^{35}$ The increase in expected procurement cost would be less pronounced if the utility chose to undertake the core project (with procurement cost 100,000) rather than the non-core project. The utility would secure zero profit under both projects.

${ }^{36}$ Multiple optional compensation structures may also be rare in practice because of their perceived complexity and because regulators may lack the knowledge required to structure options optimally.

${ }^{37}$ A DER can be owned, installed, and operated by the utility. For instance, in 2014, Arizona Public Service was approved to own, install, and operate $10 \mathrm{MWs}$ of rooftop solar capacity (ACC, 2014). Alternatively, a
} 
required to undertake only external DER projects (NYPSC, 2014a, 2015, 2016). A question of interest is whether the associated financing arrangements should vary systematically from the corresponding arrangements for internal DER projects.

Because external DER projects can arise from many diverse, innovative sources, they may often entail relatively low expected cost while introducing considerable potential cost variation. Also, because utilities may be relatively unfamiliar with the design and implementation of external DER projects, a utility's cost-containment effort may be relatively ineffective and/or onerous.

Our findings suggest that more cost sharing often is implemented when $\theta=\theta_{1}$ (and also when $\theta=\theta_{2}$ at the solution to [RP1]): (i) as the non-core project entails greater potential cost variation $(\bar{c}-\underline{c})$ and lower expected cost (e.g., a smaller value of $\phi_{1}$ ); (ii) as the efficacy of the utility's cost reducing effort declines (e.g., as $\alpha$ declines); and (iii) as cost-containment effort becomes more onerous for the utility to deliver (e.g., as $\delta$ increases). Consequently, our findings suggest that the optimal extent of cost sharing will often be higher when the non-core project is an external project than when it is an internal project.

To assess the extent to which cost sharing may optimally increase as internal DER projects are replaced by external projects, suppose the environment with an internal noncore project is as specified in the baseline setting. Further suppose that the environment with an external non-core project differs only in that $\underline{c}=30,000, \bar{c}=130,000, \alpha=0.3$, and $\delta=75$. Thus, relative to the internal non-core project, the external non-core project entails lower cost realizations $(\underline{c}$ and $\bar{c})$ and the utility's cost-containment effort is less effective and more onerous. The selected parameters ensure that the efficient expected full cost is similar under the internal and external non-core projects. ${ }^{38}$

utility can procure a DER project (e.g., rooftop solar capacity) from an external third-party vendor that is not affiliated with the utility. External projects often are encouraged to stimulate the development of a competitive supply of DER projects and to limit the ability of utilities to exercise any market power that they might possess. Regulators in California and New York require nearly all DER projects to be external (NYPSC, 2014a, 2015, 2016; CPUC, 2016b).

${ }^{38}$ Efficient expected full cost is $\phi_{1} E c_{1}^{*}+\phi_{2} E c_{2}^{*}$, where $E c_{i}^{*}$ is defined in expression (6). In the present setting, the efficient expected full cost is 88,233 under the internal non-core project and $86,842(1.6 \%$ lower) under the external non-core project. 
Table 3 records selected elements of the solution to $[\mathrm{RP}]$ in this setting. The second (third) column in the table presents outcomes under the optimal procurement policy when the non-core project is internal (external). In both cases, the utility always undertakes the non-core project. Table 3 reports that the regulator optimally implements considerably more cost sharing when $\theta=\theta_{1}$ if the non-core project is external than if it is internal. The incremental reward for $\underline{c}$ rather than $\bar{c}$ (i.e., $\Delta_{1}$ ) is $14.1 \%$ higher when the non-core project is internal than when it is external.

\begin{tabular}{|c|c|c|c|}
\hline & $\begin{array}{c}\text { Internal Project: } \\
\text { Optimal Policy }\end{array}$ & $\begin{array}{c}\text { External Project: } \\
\text { Optimal Policy }\end{array}$ & $\begin{array}{c}\text { External Project: } \\
\text { Wrong Policy }\end{array}$ \\
\hline$\underline{r}_{1}$ & 58,625 & 40,272 & 58,625 \\
\hline $\bar{r}_{1}$ & 141,171 & 124,974 & 141,171 \\
\hline$\Delta_{1}$ & 17,454 & 15,298 & 17,454 \\
\hline$e_{1}$ & 4.94 & 3.30 & 3.48 \\
\hline$\underline{r}_{2}=\bar{r}_{2}$ & 84,199 & 82,492 & 84,199 \\
\hline$E\{\pi\}$ & 1,091 & 956 & 10,292 \\
\hline$E\{r\}$ & 90,362 & 88,686 & 97,908 \\
\hline
\end{tabular}

\section{Table 3. Outcomes under Optimal and Suboptimal Procurement Policies.}

The last column in Table 3 presents the outcomes that would arise in this setting if the regulator implemented the procurement policy that is optimal in the presence of the internal non-core project when, in fact, the project is the external one. The utility continues to always undertake the external non-core project in this setting even when the "wrong" procurement policy (i.e., the policy that is optimal in the presence of the internal non-core project) ${ }^{39}$ is implemented. However, despite the similarity of efficient expected full costs under the two non-core projects, implementation of the wrong procurement policy under the external non-core project causes expected procurement cost $(E\{r\})$ to increase by $10.4 \%$ above the ${ }^{39}$ This definition of the "wrong" procurement policy is maintained throughout the ensuing discussion. 
level achieved under the optimal policy, and allows the utility's profit to increase more than ten-fold. These outcomes arise in part because the wrong policy effectively compensates the utility for cost-containment effort that it does not deliver (because $\alpha$ is relatively low and $\delta$ is relatively high under the external non-core project).

Procurement costs can increase even further if implementation of the wrong policy induces the utility to choose an unintended project. To illustrate, suppose the environment coincides with the setting of Table 3 with the exception that under the external non-core project, the environment is as specified in the baseline setting except that $\underline{c}=40,000, \theta_{2}=0.80$, $\phi_{1}=0.15, \alpha=0.25$, and $\delta=75$. These parameters are chosen to ensure the utility's costcontainment effort is less effective and more onerous under the external non-core project than under the internal project, but efficient expected full costs are nearly identical under the two projects. ${ }^{40}$ Because the external project is relatively unlikely to have low potential $\left(\phi_{1}=0.15\right)$, the optimal procurement policy induces the utility to undertake the core project when $\theta=\theta_{1}$, thereby eliminating the utility's rent. ${ }^{41}$ However, when the wrong policy is implemented, the utility always undertakes the non-core project and selects the $\left(\underline{r}_{1}, \bar{r}_{1}\right)$ cost-sharing plan. ${ }^{42}$ Implementation of the wrong policy in this setting increases expected procurement cost by $13.2 \%$ above the level that the optimal policy would secure in the presence of the external non-core project. ${ }^{43}$

Of course, procurement costs can increase even more dramatically if the external non-core project has the potential to deliver substantially lower cost but the procurement policy fails to capture the potential cost reduction for consumers. To illustrate, suppose the environment coincides with the setting of Table 3 except that under the external non-core project, the

${ }^{40}$ The efficient expected full cost in this environment is 88,233 under the internal non-core project and 88, 288 (approximately 0.06\% higher) under the external non-core project.

${ }^{41}$ Under the optimal policy in the presence of the external non-core project, $\underline{r}_{2}=\bar{r}_{2}=78,962$ and expected procurement cost is $\phi_{1}[100,000]+\phi_{2}[78,962]=82,118$.

${ }^{42} \underline{r}_{1}=58,625, \bar{r}_{1}=141,171$, and $\underline{r}_{2}=\bar{r}_{2}=84,199$ under the optimal policy when the non-core project is known to be an internal project.

${ }^{43}$ The utility's expected profit under the wrong policy is 5,998 , which is approximately $6.4 \%$ of expected procurement cost $(92,994)$. 
environment reflects the baseline setting but with $\underline{c}=20,000, \bar{c}=120,000, \theta_{1}=0.50$, $\theta_{2}=0.75, \phi_{1}=0.10$, and $\alpha=0.45$. The efficient expected full cost is nearly $50 \%$ lower in this environment under the external non-core project than under the internal project. ${ }^{44}$ Consequently, if the regulator implements the policy that is optimal in the presence of the internal non-core project when the non-core project is actually the external project, expected procurement cost increases by $82.9 \%$ and the utility's expected profit increases by nearly $7,000 \%$ above the corresponding levels that arise under the optimal procurement policy. ${ }^{45}$

\section{Alternative Environment.}

Before concluding, we briefly consider an alternative environment in which the efficacy of the utility's cost-containment effort is less pronounced, rather than more pronounced, when the non-core project has high potential (so $p_{e}\left(e, \theta_{1}\right)>p_{e}\left(e, \theta_{2}\right)$ for all $e \geq 0$ ). Such an environment prevails when $e$ and $\theta$ are substitutes rather than complements, so it is relatively difficult for the utility to reduce expected cost under the non-core project when innate cost is relatively low. Conclusions 11 and 12 report two new qualitative conclusions that can arise in this environment. ${ }^{46}$

Conclusion 11. Suppose $p_{e}\left(e, \theta_{1}\right)>p_{e}\left(e, \theta_{2}\right)$ for all $e \geq 0$ and $p\left(e_{1}^{*}, \theta_{1}\right)=p\left(e_{2}^{*}, \theta_{2}\right)$. Then $\bar{r}_{1}=\underline{r}_{1}=\bar{r}_{2}=\underline{r}_{2}=\bar{c}+D\left(e_{1}^{*}\right)-p\left(e_{1}^{*}, \theta_{1}\right)[\bar{c}-\underline{c}]$ at the solution to [RP].

When $e$ and $\theta$ are substitutes, the low cost realization $(\underline{c})$ can be equally likely for the two realizations of $\theta$ when the utility delivers the efficient level of cost-containment effort. In this event, reducing $\Delta_{1}$ below $\bar{c}-\underline{c}$ (and increasing $\bar{r}_{1}$ to leave $\pi_{1}^{n}\left(\theta_{1}\right)$ unchanged) will not

\footnotetext{
${ }^{44}$ The efficient expected full cost in this setting is 88,233 under the internal non-core project and 45,275 (48.7\% lower) under the external non-core project.

${ }^{45}$ When the non-core project is the external project, $\underline{r}_{1}=22,271, \bar{r}_{1}=117,312, \underline{r}_{2}=\bar{r}_{2}=44,646$, expected procurement cost is 46,352, and the utility's expected profit is 559 under the optimal policy in this environment. When the wrong policy is implemented, expected procurement cost is 84,764 and the utility's expected profit is 39,302 .

${ }^{46}$ For brevity and expositional clarity, the analysis in this section focuses on settings where $p\left(e_{i}^{*}, \theta_{i}\right) \underline{c}+$ $\left[1-p\left(e_{i}^{*}, \theta_{i}\right)\right] \bar{c}+D\left(e_{i}^{*}\right)$ is sufficiently small relative to $c_{0}$ for $i=1,2$ that the regulator always induces the utility to undertake the non-core project.
} 
reduce $\pi_{1}^{n}\left(\theta_{2}\right)$, the profit the utility anticipates by understating $\theta_{2} \cdot{ }^{47}$ If the regulator cannot reduce expected procurement cost when $\theta=\theta_{2}$ by reducing $\Delta_{1}$ below $\bar{c}-\underline{c}$, then she will set both $\Delta_{1}$ and $\Delta_{2}$ equal to $\bar{c}-\underline{c}$ by offering the utility a single fixed payment for undertaking the non-core project. ${ }^{48}$

Conclusion 12. When $p_{e}\left(e, \theta_{2}\right)<p_{e}\left(e, \theta_{1}\right)$ for all $e \geq 0$, the utility may be rewarded with more than the full cost reduction it achieves (i.e., $\underline{r}_{1}>\bar{r}_{1}$ and so $\Delta_{1}>\bar{c}-\underline{c}$ ) at the solution to $[R P]$.

Recall that the regulator may optimally allow $\Delta_{1}$ to diverge from $\bar{c}-\underline{c}$ in order to reduce $\pi_{1}^{n}\left(\theta_{2}\right)$. When $e$ and $\theta$ are substitutes and $p\left(e_{1}^{*}, \theta_{1}\right)>p\left(e_{2}^{*}, \theta_{2}\right), \pi_{1}^{n}\left(\theta_{2}\right)$ declines when $\Delta_{1}$ is increased above $\bar{c}-\underline{c}$ and $\bar{r}_{1}$ is reduced so as to leave $\pi_{1}^{n}\left(\theta_{1}\right)$ unchanged. The reduction in $\pi_{1}^{n}\left(\theta_{2}\right)$ arises because cost-containment effort is less effective at reducing expected cost when $\theta=\theta_{2}$ than when $\theta=\theta_{1}$. Conclusion 12 implies that if the efficacy of cost-containment effort is sufficiently more pronounced when innate cost is high than when it is low, then the optimal procurement policy can effectively reward the utility more than the full amount of the cost saving that is achieved when $\underline{c}$ rather than $\bar{c}$ is realized.

Together, Conclusions 2, 11, and 12 imply that although the optimal procurement policy typically entails some cost sharing $\left(\Delta_{1}<\bar{c}-\underline{c}\right)$ when $e$ and $\theta$ are complements, cost sharing may not be optimal when $e$ and $\theta$ are substitutes. Consequently, an important initial step in designing procurement policy is to determine whether cost-containment effort is more effective when innate costs are low or high. ${ }^{49}$

$\left.\overline{{ }^{47} \text { Recall from Lemma } 2 \text { that } \frac{\partial}{\partial \Delta_{1}}\left\{\pi_{1}^{n}\left(\theta_{2}\right)\right.}-\pi_{1}^{n}\left(\theta_{1}\right)\right\}=p\left(e_{12}, \theta_{2}\right)-p\left(e_{1}, \theta_{1}\right)=p\left(e_{2}^{*}, \theta_{2}\right)-p\left(e_{1}^{*}, \theta_{1}\right)$ when $\Delta_{1}=\bar{c}-\underline{c}$.

${ }^{48}$ This fixed payment ensures $\pi_{1}^{n}\left(\theta_{1}\right)=0$ when $e_{1}=e_{1}^{*}$.

${ }^{49}$ If cost-containment effort is determined to be relatively effective when innate cost is high, then it is important to assess the extent to which $e$ and $\theta$ are substitutes. If $p_{e}\left(e, \theta_{1}\right)-p_{e}\left(e, \theta_{2}\right)$ is positive but sufficiently small for all $e \geq 0$, then the qualitative conclusions drawn in sections 4 and 5 will prevail. 


\section{Conclusions}

We have analyzed the optimal design of policies to encourage electricity distribution utilities to adopt efficient DER projects and to manage the costs of these projects. Our findings support calls for the implementation of performance based regulation (PBR) that requires utilities to share DER cost savings and cost overruns with their customers (NYPSC, 2015, 2016; Lowry et al., 2016). We found that an optimally-designed PBR policy often can reduce expected procurement cost well below the level secured when the utility's realized cost is fully reimbursed. We also demonstrated that the optimal policy can vary considerably with the environment in which it is implemented. For instance, more extensive cost sharing often is advisable in the presence of more pronounced information asymmetry regarding the costs that are likely to prevail under a DER project. We also showed that procurement costs can increase substantially if the compensation structure that is implemented is not adequately tailored to the prevailing environment. In addition, we found that although the introduction of multiple optional compensation policies typically does not reduce procurement costs dramatically, it can substantially reduce the utility's rent.

We have focused on a simple environment in order to illustrate most clearly the key qualitative properties of optimal compensation structures. These properties persist more generally. For instance, as the analysis in the Appendix demonstrates, these properties persist when the potential of new DER projects $(\theta)$ can assume many distinct values, rather than only two values. The optimal policy in this setting when $e$ and $\theta$ are complements continues to include cost sharing and a bias toward the core project in order to limit the utility's rent under the non-core project. Furthermore, although procurement costs often do not rise substantially when the regulator does not offer the utility a choice among reward structures, utility profit can increase dramatically. ${ }^{50}$

Our streamlined model was designed to include several important elements of the envi-

\footnotetext{
${ }^{50}$ The primary qualitative change that arises when $\theta$ can take on any one of a continuum of values is that the menu of optional compensation structures typically will consist entirely of cost-sharing plans rather than a cost sharing plan and a fixed payment.
} 
ronments in which regulators routinely operate. Future research should expand our analysis to include other important elements of the regulatory environment. For instance, risk aversion merits explicit consideration. Fixed payments to the utility typically will not be optimal if the utility is more averse to risk than are consumers. More generally, the optimal extent of cost sharing will vary with the relative risk aversion of the utility and consumers.

Future research also should allow the utility to choose among multiple core, internal non-core, and external non-core projects. In such a setting, a bias toward either internal or external non-core projects may optimally be induced, depending on the relative severity of the adverse selection and moral hazard problems the regulator faces. In addition, future research should examine how compensation for DER projects is optimally integrated with other relevant elements of industry policy, including DER tax credits, the treatment of DER capacity in capacity auctions, revenue decoupling, and other components of retail rate design. 


\section{Appendix}

Part A of this Appendix reports how $\Delta$ changes at the solution to [RP1] as model parameters change. Part B presents additional findings. Part $\mathrm{C}$ provides the proofs of the Conclusions in the text whose proofs do no not follow immediately from the discussion in the text.

\section{A. Comparative Statics when Optional Cost-Sharing Plans are Not Feasible.}

Conclusions $13-17$ pertain to the setting where $c_{0}-E c_{1}^{*}$ is sufficiently large that the regulator always induces the utility to implement the non-core project. ${ }^{51}$

Conclusion 13. Suppose $p_{e}\left(e, \theta_{2}\right)-p_{e}\left(e, \theta_{1}\right)$ is sufficiently close to 0 for all $e \geq 0$. Then $\frac{d \Delta}{d \phi_{1}}>0$ at the solution to [RP1].

Conclusion 14. At the solution to [RP1]: (i) $\frac{d \Delta}{d \theta_{2}}<0$ if $p_{e \theta}\left(e, \theta_{2}\right) \geq 0$ and $\left|p_{e e \theta}\left(e, \theta_{2}\right)\right|$ are sufficiently small for all $e \geq 0$; (ii) $\frac{d \Delta}{d \theta_{1}}>0$ if $\phi_{2}$ is sufficiently large or $\left|p_{e e}\left(e, \theta_{1}\right)\right|$ is sufficiently small for all $e \geq 0$; and so (iii) $\frac{d \Delta}{d\left(\theta_{2}-\theta_{1}\right)}<0$ if $p_{e \theta}\left(e, \theta_{2}\right),\left|p_{e e}\left(e, \theta_{1}\right)\right|$, and $\left|p_{\text {ee } \theta}\left(e, \theta_{2}\right)\right|$ are sufficiently small for all $e \geq 0$.

Conclusion 15. $\quad \frac{d(\underline{r}-\bar{r})}{d(\bar{c}-\underline{c})}<0$ and $\frac{d \Delta}{d(\bar{c}-\underline{c})}<1$ at the solution to [RP1].

Conclusion 16. At the solution to [RP1]: (i) $\frac{d \Delta}{d \delta_{2}}>0$; and (ii) $\frac{d \Delta}{d \delta_{1}}>0$ when $\phi_{1}$ is sufficiently large whereas $\frac{d \Delta}{d \delta_{1}}<0$ when $\phi_{2}$ is sufficiently large. Therefore, $\frac{d \Delta}{d \delta}>0$ when $\phi_{1}$ is sufficiently large, where $\delta=\delta_{1}=\delta_{2}$.

Conclusion 17. If $p_{e}\left(e, \theta_{1}\right)=p_{e}\left(e, \theta_{2}\right)=\alpha>0$ for all $e \geq 0$, then $\frac{d \Delta}{d \alpha}>0$ at the solution to [RP1].

\section{B. Additional Findings.}

In order to identify the key elements of the optimal DER procurement policy most clearly, the analysis in the text focused on the relatively simple setting where the project potential $(\theta)$ could assume one of only two values $\left(\theta_{1}\right.$ or $\left.\theta_{2}\right)$. Now consider the more realistic setting where $\theta$ can assume any value in the interval $[\underline{\theta}, \bar{\theta}]$. Suppose that $E \bar{c}^{*} \equiv \min _{e}\{p(e, \bar{\theta}) \underline{c}+$ $[1-p(e, \bar{\theta})] \bar{c}+D(e)\}<c_{0}$. Further suppose that $E \underline{c}^{*} \equiv \min _{e}\{p(e, \underline{\theta}) \underline{c}+[1-p(e, \underline{\theta})] \bar{c}+$ $D(e)\}>c_{0}$ and $E \underline{c}^{*}-c_{0}$ is sufficiently large that the regulator optimally induces the utility

\footnotetext{
${ }^{51}$ Brown and Sappington $(2017 \mathrm{~b})$ provide the proofs of these Conclusions, which parallel the proofs of
} Conclusions $3-7$ below. 
to undertake the non-core project for some, but not all, realizations of $\theta \in[\underline{\theta}, \bar{\theta}]$. In addition, suppose $p_{e \theta}(e, \theta) \geq 0$ for all $e \geq 0$ and $\theta \in[\underline{\theta}, \bar{\theta}]$.

Let $[\mathrm{RPC}]$ denote the regulator's problem in this setting when only a single compensation policy for the non-core project is feasible. Also let $\widehat{\theta} \in(\underline{\theta}, \bar{\theta})$ denote the realization of $\theta$ for which the utility secures the same expected profit under the core project and the noncore project. In addition, let $e(\theta)$ denote the amount of cost-containment effort the utility supplies under the non-core project when $\theta$ is realized. Conclusion 18 characterizes the optimal procurement policy in this setting. ${ }^{52}$

Conclusion 18. At the solution to [RPC]: (i) $r_{0}=c_{0}$; (ii) $\underline{r}<\bar{r}$ and $\underline{r}-\underline{c}>\bar{r}-\bar{c}$; (iii) the utility undertakes the core project for all $\theta \in[\underline{\theta}, \widehat{\theta})$ and undertakes the non-core project for all $\theta \in[\widehat{\theta}, \bar{\theta}]$; (iv) the utility's expected profit is strictly increasing in $\theta$ for all $\theta \in[\widehat{\theta}, \bar{\theta})$; and $(v) p(e(\widehat{\theta}), \widehat{\theta}) \underline{c}+[1-p(e(\widehat{\theta}), \widehat{\theta})] \bar{c}+D(e(\widehat{\theta}))<c_{0}$.

Conclusion 18 reports that the regulator induces the utility to implement the core project and earn no rent for the smaller realizations of $\theta$. Because the utility's expected profit declines as $\theta$ increases whenever $\underline{r}-\underline{c}>\bar{r}-\bar{c}$, the utility secures rent for all $\theta \in(\hat{\theta}, \bar{\theta}]$ when it implements the non-core project. The regulator implements cost sharing $(\underline{r}<\bar{r})$ to reduce this rent. To further reduce the utility's rent under the non-core project, the regulator induces the utility to undertake the core project even when doing so does not minimize industry costs (as reflected in property (v) of Conclusion 18).

It can also be shown that if the regulator can offer the utility a choice between two distinct compensation structures when it implements the non-core project, both structures generally will entail nontrivial cost sharing $(\underline{r}<\bar{r})$. More (less) pronounced cost sharing will be implemented for the smaller (larger) $\theta$ realizations for which the non-core project is undertaken. The cost sharing reduces the rate at which the utility's rent increases with $\theta$, and thereby reduces expected procurement cost. Thus, the key qualitative conclusion in the text that is an artifact of the assumption that $\theta$ is binary is the fixed payment that the utility accepts under the non-core project when $\theta=\theta_{2}$. When $\theta \in[\underline{\theta}, \bar{\theta}]$, the regulator typically will only include a fixed payment in the set of optional compensation structures if she can offer a continuum of such structures.

As in the binary setting considered in the text, the ability to offer two distinct costsharing compensation structures rather than only one often enables the regulator to reduce the utility's expected profit substantially, but typically does not permit a major reduction in expected procurement cost. To illustrate, consider the baseline setting with the exception that $\theta$ has a uniform distribution on $[0,0.75]$. In this setting, expected procurement cost is 90,795 and the utility's expected profit is 4,300 at the solution to [RPC]. When the regulator can offer two distinct cost-sharing compensation structures, she is able to reduce expected procurement cost (by 0.15\%) to 90,655 and the utility's expected profit (by 69\%) to 1,339.

\footnotetext{
${ }^{52}$ The proof of Conclusion 18 is provided in Brown and Sappington (2017b).
} 


\section{Proofs of Conclusions in the Text.}

Proof of Lemma 2. When $\Delta_{i}>0$ :

$$
\begin{aligned}
\pi_{i}^{n}\left(\theta_{2}\right) & =\bar{r}_{i}-\bar{c}+p\left(e_{i 2}, \theta_{2}\right) \Delta_{i}-D\left(e_{i 2}\right) \geq \bar{r}_{i}-\bar{c}+p\left(e_{i 1}, \theta_{2}\right) \Delta_{i}-D\left(e_{i 1}\right) \\
& >\bar{r}_{i}-\bar{c}+p\left(e_{i 1}, \theta_{1}\right) \Delta_{i}-D\left(e_{i 1}\right)=\pi_{i}^{n}\left(\theta_{1}\right) .
\end{aligned}
$$

Furthermore, the utility's profit-maximizing choice of effort implies that when $\Delta_{j}>0$ :

$$
\begin{gathered}
p_{e}\left(e_{j i}, \theta_{i}\right) \Delta_{j}=D^{\prime}\left(e_{j i}\right) \Rightarrow \frac{d e_{j i}}{d \underline{r}_{j}}=-\frac{d e_{j i}}{d \bar{r}_{j}}=-\frac{p_{e}\left(e_{j i}, \theta_{i}\right)}{\Delta_{j}\left[p_{e e}\left(e_{j i}, \theta_{i}\right) \Delta_{j}-D^{\prime \prime}\left(e_{j i}\right)\right]}>0, \\
\text { and } \frac{d e_{j i}}{d \theta_{i}}=-\frac{p_{e \theta}\left(e_{j i}, \theta_{i}\right) \Delta_{j}}{p_{e e}\left(e_{j i}, \theta_{i}\right) \Delta_{j}-D^{\prime \prime}\left(e_{j i}\right)} \geq 0 .
\end{gathered}
$$

(7), (8), and the envelope theorem imply:

$$
\frac{\partial}{\partial \Delta_{i}}\left\{\pi_{i}^{n}\left(\theta_{2}\right)-\pi_{i}^{n}\left(\theta_{1}\right)\right\}=p\left(e_{i 2}, \theta_{2}\right)-p\left(e_{i 1}, \theta_{1}\right)>0 .
$$

Proof of Conclusion 2. The regulator's problem, [RP], is:

$$
\begin{aligned}
\underset{\psi_{i} \in\{0,1\}, \underline{r}_{i}, \bar{r}_{i}}{\operatorname{Maximize}} & -\phi_{1} \psi_{1}\left\{p\left(e_{1}, \theta_{1}\right) \underline{r}_{1}+\left[1-p\left(e_{1}, \theta_{1}\right] \bar{r}_{1}\right\}\right. \\
& -\phi_{2} \psi_{2}\left\{p\left(e_{2}, \theta_{2}\right) \underline{r}_{2}+\left[1-p\left(e_{2}, \theta_{2}\right] \bar{r}_{2}\right\}-\left[1-\phi_{1} \psi_{1}-\phi_{2} \psi_{2}\right] r_{0}\right.
\end{aligned}
$$

subject to, for $i, j \in\{1,2\}(j \neq i)$ :

$$
\begin{gathered}
\psi_{i}\left\{p\left(e_{i}, \theta_{i}\right)\left[\underline{r}_{i}-\underline{c}\right]+\left[1-p\left(e_{i}, \theta_{i}\right)\right]\left[\bar{r}_{i}-\bar{c}\right]-D\left(e_{i}\right)\right\} \\
+\left[1-\psi_{i}\right]\left[r_{0}-c_{0}\right] \geq 0 ; \text { and } \\
\begin{aligned}
\psi_{i}\left\{p\left(e_{i}, \theta_{i}\right)\left[\underline{r}_{i}-\underline{c}\right]+\left[1-p\left(e_{i}, \theta_{i}\right)\right]\left[\bar{r}_{i}-\bar{c}\right]-D\left(e_{i}\right)\right\}+\left[1-\psi_{i}\right]\left[r_{0}-c_{0}\right] \\
\geq \psi_{j}\left\{p\left(e_{j i}, \theta_{i}\right)\left[\underline{r}_{j}-\underline{c}\right]+\left[1-p\left(e_{j i}, \theta_{i}\right)\right]\left[\bar{r}_{j}-\bar{c}\right]-D\left(e_{j i}\right)\right\} \\
+\left[1-\psi_{j}\right]\left[r_{0}-c_{0}\right] .
\end{aligned}
\end{gathered}
$$

Let $\lambda_{i}$ and $\lambda_{i j}$ denote the Lagrange multipliers associated with constraints (10) and (11), respectively. Also let $[\mathrm{RP}]^{\prime}$ denote problem $[\mathrm{RP}]$ with constraint (10) omitted for $i=2$ and constraint (11) omitted for $i=1$. We will show that the solution to [RP $]^{\prime}$ is a feasible solution to $[\mathrm{RP}]$, and so must be a solution to $[\mathrm{RP}]$.

The necessary conditions for a solution to $[\mathrm{RP}]^{\prime}$ include the following:

$$
r_{0}: \quad-1+\phi_{1} \psi_{1}+\phi_{2} \psi_{2}+\lambda_{1}\left[1-\psi_{1}\right]+\lambda_{21}\left[1-\psi_{2}\right]-\lambda_{21}\left[1-\psi_{1}\right]=0 ;
$$


$\underline{r}_{1}: \quad \psi_{1}\left\{-\phi_{1} p\left(e_{1}, \theta_{1}\right)-\phi_{1} p_{e}\left(e_{1}, \theta_{1}\right)\left[\underline{r}_{1}-\bar{r}_{1}\right] \frac{d e_{1}}{d \underline{r}_{1}}\right.$

$$
\left.+\lambda_{1} p\left(e_{1}, \theta_{1}\right)-\lambda_{21} p\left(e_{12}, \theta_{2}\right)\right\}=0
$$

$\bar{r}_{1}: \quad \psi_{1}\left\{-\phi_{1}\left[1-p\left(e_{1}, \theta_{1}\right)\right]-\phi_{1} p_{e}\left(e_{1}, \theta_{1}\right)\left[\underline{r}_{1}-\bar{r}_{1}\right] \frac{d e_{1}}{d \bar{r}_{1}}\right.$

$$
\left.+\lambda_{1}\left[1-p\left(e_{1}, \theta_{1}\right)\right]-\lambda_{21}\left[1-p\left(e_{12}, \theta_{2}\right)\right]\right\}=0
$$

$\underline{r}_{2}: \quad \psi_{2}\left\{-\phi_{2} p\left(e_{2}, \theta_{2}\right)-\phi_{2} p_{e}\left(e_{2}, \theta_{2}\right)\left[\underline{r}_{2}-\bar{r}_{2}\right] \frac{d e_{2}}{d \underline{r}_{2}}+\lambda_{21} p\left(e_{2}, \theta_{2}\right)\right\}=0 ;$

$\bar{r}_{2}: \quad \psi_{2}\left\{-\phi_{2}\left[1-p\left(e_{2}, \theta_{2}\right)\right]-\phi_{2} p_{e}\left(e_{2}, \theta_{2}\right)\left[\underline{r}_{2}-\bar{r}_{2}\right] \frac{d e_{2}}{d \bar{r}_{2}}\right.$

$$
\left.+\lambda_{21}\left[1-p\left(e_{2}, \theta_{2}\right)\right]\right\}=0 .
$$

Summing (13) and (14) and employing (8) provides:

$$
\psi_{1}\left[\lambda_{1}-\phi_{1}-\lambda_{21}\right]=0 .
$$

Summing (15) and (16) and employing (8) provides:

$$
\psi_{2}\left[\lambda_{21}-\phi_{2}\right]=0
$$

(18) implies that $\lambda_{21}=\phi_{2}$ because $\psi_{2}>0$ by assumption. Therefore, $\pi_{2}\left(\theta_{2}\right)=\pi_{1}\left(\theta_{2}\right)$. Furthermore, because $\lambda_{21}=\phi_{2}$ and $\psi_{1}>0$, (17) implies $\lambda_{1}=1$, and so $\pi_{1}\left(\theta_{1}\right)=0$.

Because $\lambda_{21}=\phi_{2}$ and $\psi_{2}=1,(8)$ and (15) imply:

$$
\phi_{2} p_{e}\left(e_{2}, \theta_{2}\right)\left[\underline{r}_{2}-\bar{r}_{2}\right] \frac{d e_{2}}{d \underline{r}_{2}}=0 \quad \Rightarrow \quad \underline{r}_{2}=\bar{r}_{2}
$$

Because $\lambda_{1}=1, \lambda_{21}=\phi_{2}$, and $\psi_{1}=1,(13)$ implies:

$$
\phi_{2}\left[p\left(e_{12}, \theta_{2}\right)-p\left(e_{1}, \theta_{1}\right)\right]=\phi_{1} p_{e}\left(e_{1}, \theta_{1}\right)\left[\bar{r}_{1}-\underline{r}_{1}\right] \frac{d e_{1}}{d \underline{r}_{1}} \text {. }
$$

(8) and (19) imply that $\bar{r}_{1}-\underline{r}_{1}>0$ because $p\left(e_{12}, \theta_{2}\right)>p\left(e_{1}, \theta_{1}\right)$ under the maintained assumptions.

It remains to prove that the solution to $[\mathrm{RP}]^{\prime}$ is the solution to $[\mathrm{RP}]$. To do so, it suffices to show that at the solution to [RP] $]^{\prime},(10)$ holds for $i=2$ and (11) holds for $i=1$ and $j=2$.

Because $\lambda_{1}>0, \lambda_{21}>0, \psi_{2}=1$, and $\underline{r}_{2}=\bar{r}_{2}=r_{2}$ at the solution to $[\mathrm{RP}]^{\prime},(10)$ and (11) imply:

$$
\pi_{1}^{n}\left(\theta_{1}\right)=0 \text { and } r_{2}=p\left(e_{2}^{*}, \theta_{2}\right) \underline{c}+\left[1-p\left(e_{2}^{*}, \theta_{2}\right)\right] \bar{c}+D\left(e_{2}^{*}\right)+\pi_{1}^{n}\left(\theta_{2}\right) .
$$

(20) and Lemma 2 imply $\pi_{2}^{n}\left(\theta_{2}\right)=\pi_{1}^{n}\left(\theta_{2}\right)>\pi_{1}^{n}\left(\theta_{1}\right)=0$. Therefore, (10) holds for $i=2$. 
$e_{21}=e_{1}^{*}$ because $\psi_{2}=1$ and $\underline{r}_{2}=\bar{r}_{2}=r_{2}$ at the solution to $[\mathrm{RP}]^{\prime}$. Therefore, (20) implies that the utility's expected profit when $\theta=\theta_{1}$ and it reports $\theta=\theta_{2}$ is:

$$
\begin{aligned}
r_{2}- & \left\{p\left(e_{1}^{*}, \theta_{1}\right) \underline{c}+\left[1-p\left(e_{1}^{*}, \theta_{1}\right)\right] \bar{c}+D\left(e_{1}^{*}\right)\right\} \\
= & p\left(e_{2}^{*}, \theta_{2}\right) \underline{c}+\left[1-p\left(e_{2}^{*}, \theta_{2}\right)\right] \bar{c}+D\left(e_{2}^{*}\right) \\
& \quad-\left\{p\left(e_{1}^{*}, \theta_{1}\right) \underline{c}+\left[1-p\left(e_{1}^{*}, \theta_{1}\right)\right] \bar{c}+D\left(e_{1}^{*}\right)\right\}+\pi_{1}^{n}\left(\theta_{2}\right) \\
= & r_{2}-\left\{p\left(e_{1}^{*}, \theta_{1}\right) \underline{c}+\left[1-p\left(e_{1}^{*}, \theta_{1}\right)\right] \bar{c}+D\left(e_{1}^{*}\right)\right\} \\
& \quad-\left(r_{2}-\left\{p\left(e_{2}^{*}, \theta_{2}\right) \underline{c}+\left[1-p\left(e_{2}^{*}, \theta_{2}\right)\right] \bar{c}+D\left(e_{2}^{*}\right)\right\}\right)+\pi_{1}^{n}\left(\theta_{2}\right)-\pi_{1}^{n}\left(\theta_{1}\right) \\
= & \pi_{1}^{n}\left(\theta_{2}\right)-\pi_{1}^{n}\left(\theta_{1}\right)-\left[\pi_{2}^{n}\left(\theta_{2}\right)-\pi_{2}^{n}\left(\theta_{1}\right)\right] \leq 0 .
\end{aligned}
$$

The equality in (21) holds because $\pi_{1}^{n}\left(\theta_{1}\right)=0$. The inequality in (22) follows from Lemma 2 because $\Delta_{2}=\bar{c}-\underline{c}>\Delta_{1}$. (22) ensures that (11) holds for $i=1$ and $j=2$.

Proof of Lemma 3. (12), (17), and (18) imply:

$$
\begin{aligned}
1-\lambda_{1} & =\phi_{1} \psi_{1}+\phi_{2} \psi_{2}-\lambda_{1} \psi_{1}+\lambda_{21}\left[\psi_{1}-\psi_{2}\right] \\
& =\psi_{2}\left[\phi_{2}-\lambda_{21}\right]-\psi_{1}\left[\lambda_{1}-\phi_{1}-\lambda_{21}\right]=0 \Rightarrow \lambda_{1}=1 .
\end{aligned}
$$

Suppose $\psi_{1}=\psi_{2}=0$. Then $r_{0}=c_{0}$ from (10) because $\lambda_{1}=1$. Consequently, expected procurement cost is $c_{0}$.

Suppose instead that $\psi_{2}=1, \psi_{1}=0, r_{0}=c_{0}$, and $\underline{r}_{2}=\bar{r}_{2}=E c_{2}^{*}<c_{0}$. It is readily verified that this policy satisfies all of the relevant constraints in $[R P]^{\prime}$. Furthermore, expected procurement cost is $\phi_{2} \underline{r}_{2}+\phi_{1} c_{0}<c_{0}$. Consequently, expected procurement cost is strictly lower under this feasible solution to $[\mathrm{RP}]^{\prime}$, which implies it cannot be the case that $\psi_{1}=\psi_{2}=0$. Therefore, $\psi_{1}+\psi_{2}>0$.

Because $\lambda_{1}=1,(17)$ and (18) imply:

$\psi_{1}\left[\phi_{2}-\lambda_{21}\right]=0=-\psi_{2}\left[\phi_{2}-\lambda_{21}\right] \Rightarrow\left[\psi_{1}+\psi_{2}\right] \phi_{2}-\lambda_{21} \Rightarrow \lambda_{21}=\phi_{2}$.

The last equality in (24) holds because $\psi_{1}+\psi_{2}>0$.

Because $\lambda_{21}=\phi_{2},(8)$ and (15) imply that if $\psi_{2}=1$ :

$$
\phi_{2} p_{e}\left(e_{2}, \theta_{2}\right)\left[\underline{r}_{2}-\bar{r}_{2}\right] \frac{d e_{2}}{d \underline{r}_{2}}=0 \quad \Rightarrow \quad \underline{r}_{2}=\bar{r}_{2} .
$$

Because $\lambda_{1}=1$ and $\lambda_{21}=\phi_{2},(13)$ implies that if $\psi_{1}=1$ :

$$
\phi_{2}\left[p\left(e_{12}, \theta_{2}\right)-p\left(e_{1}, \theta_{1}\right)\right]=\phi_{1} p_{e}\left(e_{1}, \theta_{1}\right)\left[\bar{r}_{1}-\underline{r}_{1}\right] \frac{d e_{1}}{d \underline{r}_{1}} .
$$

(8) and (25) imply that $\bar{r}_{1}-\underline{r}_{1}>0$. 
We now prove that $\psi_{2}=1$. To do so, first suppose $\psi_{1}=0$. Then $\psi_{2}=1$ since $\psi_{1}+\psi_{2}>0$

Now suppose $\psi_{1}=1$. Then expected procurement cost must be lower when $\theta=\theta_{1}$ if the non-core project is implemented than if the core project is implemented, i.e.:

$$
p\left(e_{1}, \theta_{1}\right) \underline{r}_{1}+\left[1-p\left(e_{1}, \theta_{1}\right)\right] \bar{r}_{1} \leq c_{0} .
$$

Arguments analogous to those employed to prove that $\psi_{1}+\psi_{2}>0$ are readily employed to demonstrate that if (26) did not hold, the regulator could secure strictly lower expected procurement cost by setting $\psi_{1}=0$.

If $\psi_{2}=0$, then expected procurement cost when $\theta=\theta_{2}$ is $c_{0}$. In contrast, if $\psi_{2}=1$, $\underline{r}_{2}=\underline{r}_{1}$, and $\bar{r}_{2}=\bar{r}_{1}$, then expected procurement cost when $\theta=\theta_{2}$ is:

$$
\begin{aligned}
p\left(e_{2}, \theta_{2}\right) \underline{r}_{2} & +\left[1-p\left(e_{2}, \theta_{2}\right)\right] \bar{r}_{2}=p\left(e_{12}, \theta_{2}\right) \underline{r}_{1}+\left[1-p\left(e_{12}, \theta_{2}\right)\right] \bar{r}_{1} \\
& <p\left(e_{1}, \theta_{1}\right) \underline{r}_{1}+\left[1-p\left(e_{1}, \theta_{1}\right)\right] \bar{r}_{1} \leq c_{0} .
\end{aligned}
$$

The first inequality in (27) holds because $\underline{r}_{1}<\bar{r}_{1}$. The second inequality in (27) reflects (26). (27) implies that when $\psi_{1}=1$, expected procurement cost is lower when $\psi_{2}=1$ than when $\psi_{2}=0$.

If $\psi_{1}=0$, then Conclusion 8 implies that expected procurement cost is $\phi_{1} c_{0}+\phi_{2} E c_{2}^{*}$. If $\psi_{1}=1$, then expected procurement cost is at most $E c_{1}^{*}$, which is expected procurement cost when $\psi_{1}=\psi_{2}=1$ and $\underline{r}_{1}=\underline{r}_{2}=\bar{r}_{1}=\bar{r}_{2}=p\left(e_{1}^{*}, \theta_{1}\right) \underline{c}+\left[1-p\left(e_{1}^{*}, \theta_{1}\right)\right] \bar{c}+D\left(e_{1}^{*}\right)$. It is readily verified that this policy is a feasible solution to $[\mathrm{RP}]^{\prime}$. Therefore, expected procurement cost is lower when $\psi_{1}=1$ than when $\psi_{1}=0$ if $E c_{1}^{*}<\phi_{1} c_{0}+\phi_{2} E c_{2}^{*}$.

Finally, as in the proof of Conclusion 2, it readily verified that the solution to $[\mathrm{RP}]^{\prime}$ is a feasible solution to $[\mathrm{RP}]$, and so is a solution to $[\mathrm{RP}]$.

Proof of Corollary 1. Conclusion 1 implies that if $\psi_{1}=0$ at the solution to $[\mathrm{RP}]^{\prime}$, then expected procurement cost is $\phi_{1} c_{0}+\phi_{2} E c_{2}^{*}$. We will show that expected procurement cost always exceeds $\phi_{1} c_{0}+\phi_{2} E c_{2}^{*}$ under the specified condition if $\psi_{1}=1$.

Because $\lambda_{1}>0$ from (17), (10) implies that expected procurement cost when $\theta=\theta_{1}$ and $\psi_{1}=1$ is:

$$
p\left(e_{1}, \theta_{1}\right) \underline{r}_{1}+\left[1-p\left(e_{1}, \theta_{1}\right)\right] \bar{r}_{1}=p\left(e_{1}, \theta_{1}\right) \underline{c}+\left[1-p\left(e_{1}, \theta_{1}\right)\right] \bar{c}+D\left(e_{1}\right) \geq E c_{1}^{*} .
$$

The weak inequality in (28) holds as a strict inequality if $\Delta_{1} \neq \bar{c}-\underline{c}$.

Because $\lambda_{21}>0$ from (24), (11) implies that expected procurement cost when $\theta=\theta_{2}$ and $\psi_{1}=1$ is:

$$
\begin{aligned}
p\left(e_{2}, \theta_{2}\right) \underline{r}_{2}+ & {\left[1-p\left(e_{2}, \theta_{2}\right)\right] \bar{r}_{2} } \\
= & p\left(e_{2}, \theta_{2}\right) \underline{c}+\left[1-p\left(e_{2}, \theta_{2}\right)\right] \bar{c}+D\left(e_{2}\right) \\
& \quad+p\left(e_{12}, \theta_{2}\right)\left[\underline{r}_{1}-\underline{c}\right]+\left[1-p\left(e_{12}, \theta_{2}\right)\right]\left[\bar{r}_{1}-\bar{c}\right]-D\left(e_{12}\right)
\end{aligned}
$$




$$
\begin{aligned}
\geq & p\left(e_{2}, \theta_{2}\right) \underline{c}+\left[1-p\left(e_{2}, \theta_{2}\right)\right] \bar{c}+D\left(e_{2}\right) \\
& +p\left(e_{1}, \theta_{1}\right)\left[\underline{r}_{1}-\underline{c}\right]+\left[1-p\left(e_{1}, \theta_{1}\right)\right]\left[\bar{r}_{1}-\bar{c}\right]-D\left(e_{1}\right) \\
& =p\left(e_{2}^{*}, \theta_{2}\right) \underline{c}+\left[1-p\left(e_{2}^{*}, \theta_{2}\right)\right] \bar{c}+D\left(e_{2}^{*}\right) .
\end{aligned}
$$

The last equality in (29) holds because $\lambda_{1}>0$. The weak inequality in (29) holds as a strict inequality if $\Delta_{1}=\bar{c}-\underline{c}$. (28) and (29) imply that expected procurement cost strictly exceeds $\phi_{1} c_{0}+\phi_{2} E c_{2}^{*}$ when $\psi_{1}=1$ if $c_{0}<E c_{1}^{*}$ or if $c_{0}-E c_{1}^{*} \geq 0$ is sufficiently small. Therefore, $\psi_{1}=0$ at the solution to $[\mathrm{RP}]^{\prime}$ under this condition.

Finally, as in the proof of Conclusion 2, it readily verified that the solution to $[\mathrm{RP}]^{\prime}$ is a solution to $[\mathrm{RP}]$.

Lemma $4-7$ are employed in the proofs of Conclusions $3-7$.

Lemma 4. $\frac{d^{2} e_{1}}{d\left(\Delta_{1}\right)^{2}}<0$ for all $\Delta_{1}>0$ at the solution to $[R P]$ when $\psi_{1}=1$ if, for all $\theta$ and $e \geq 0, p_{e \theta}(e, \theta) \geq 0$ and: (i) $D^{\prime \prime \prime}(e) \geq 0$ and $p_{e e e}(e, \theta) \leq 0$; or (ii) $D^{\prime \prime \prime}(e) \leq 0, p_{e e e}(e, \theta) \geq 0$, and $\left|D^{\prime \prime \prime}(e)\right|$ and $p_{\text {eee }}(e, \theta)$ are sufficiently small.

Proof. From (8):

$$
\begin{aligned}
& \frac{d^{2} e_{1}}{d\left(\Delta_{1}\right)^{2}} \stackrel{s}{=} \Delta_{1}\left[D^{\prime \prime}\left(e_{1}\right)-\Delta_{1} p_{e e}\left(e_{1}, \theta_{1}\right)\right] \frac{d}{d \Delta_{1}}\left\{p_{e}\left(e_{1}, \theta_{1}\right)\right\} \\
& \quad-p_{e}\left(e_{1}, \theta_{1}\right)\left\{D^{\prime \prime}\left(e_{1}\right)-\Delta_{1} p_{e e}(\cdot)+\Delta_{1}\left[D^{\prime \prime \prime}\left(e_{1}\right)-\Delta_{1} p_{e e e}(\cdot)\right] \frac{d e_{1}}{d \Delta_{1}}-\Delta_{1} p_{e e}(\cdot)\right\} .
\end{aligned}
$$

$\frac{d}{d \Delta_{1}}\left\{p_{e}\left(e_{1}, \theta_{1}\right)\right\}=p_{e e}\left(e_{1}, \theta_{1}\right) \frac{d e_{1}}{d \Delta_{1}} \leq 0$. Furthermore, $D^{\prime \prime}\left(e_{1}\right) \geq 0$ and $p_{e e}\left(e_{1}, \theta_{1}\right) \leq 0$. Therefore, the expression in the first line of (30) is non-positive. The expression in the second line of (30) is strictly negative under the maintained conditions because $p_{e}(\cdot)>0$, $\frac{d e_{1}}{d \Delta_{1}}>0$ from $(8)$, and $p_{e e}(\cdot)<0$ or $D^{\prime \prime}\left(e_{1}\right)>0$.

Lemma 5. $\frac{d e_{12}}{d \Delta_{1}}>\frac{d e_{1}}{d \Delta_{1}}$ for all $\Delta_{1}>0$ at the solution to [RP] when $\psi_{1}=1$ if, for all $\theta$ and $e \geq 0, p_{e \theta}(e, \theta) \geq 0, p_{e e \theta}(e, \theta) \geq 0$, and: (i) $D^{\prime \prime \prime}(e) \leq 0$ and $p_{e e e}(e, \theta) \geq 0$; or (ii) $D^{\prime \prime \prime}(e) \geq 0, p_{\text {eee }}(e, \theta) \leq 0$, and $D^{\prime \prime \prime}(e)$ and $\left|p_{\text {eee }}(e, \theta)\right|$ are sufficiently small.

Proof. From (8):

$$
\begin{aligned}
\frac{d e_{12}}{d \Delta_{1}}>\frac{d e_{1}}{d \Delta_{1}} & \Leftrightarrow \frac{p_{e}\left(e_{12}, \theta_{2}\right)}{\Delta_{1}\left[D^{\prime \prime}\left(e_{12}\right)-\Delta_{1} p_{e e}\left(e_{12}, \theta_{2}\right)\right]}>\frac{p_{e}\left(e_{1}, \theta_{1}\right)}{\Delta_{1}\left[D^{\prime \prime}\left(e_{1}\right)-\Delta_{1} p_{e e}\left(e_{1}, \theta_{1}\right)\right]} \\
& \Leftrightarrow \frac{D^{\prime}\left(e_{12}\right)}{D^{\prime \prime}\left(e_{12}\right)-\Delta_{1} p_{e e}\left(e_{12}, \theta_{2}\right)}>\frac{D^{\prime}\left(e_{1}\right)}{D^{\prime \prime}\left(e_{1}\right)-\Delta_{1} p_{e e}\left(e_{1}, \theta_{1}\right)} .
\end{aligned}
$$


The second equivalence in (31) holds because $p_{e}\left(e_{12}, \theta_{2}\right)=\Delta_{1} D^{\prime}\left(e_{12}\right)$ and $p_{e}\left(e_{1}, \theta_{1}\right)=$ $\Delta_{1} D^{\prime}\left(e_{1}\right)$. (8) implies that $e_{12}>e_{1}$ when $p_{e \theta}(e, \theta) \geq 0$. Therefore, $D^{\prime}\left(e_{12}\right)>D^{\prime}\left(e_{1}\right)$. Consequently, the inequality in (31) holds if:

$$
\begin{aligned}
& D^{\prime \prime}\left(e_{1}\right)-\Delta_{1} p_{e e}\left(e_{1}, \theta_{1}\right) \geq D^{\prime \prime}\left(e_{12}\right)-\Delta_{1} p_{e e}\left(e_{12}, \theta_{2}\right), \text { or } \\
& D^{\prime \prime}\left(e_{12}\right)-\Delta_{1} p_{e e}\left(e_{12}, \theta_{2}\right)-\left\{D^{\prime \prime}\left(e_{1}\right)-\Delta_{1} p_{e e}\left(e_{1}, \theta_{1}\right)\right\}>0 \text { is sufficiently small. }
\end{aligned}
$$

When $e_{12}>e_{1}$ and $p_{e e \theta}(e, \theta) \geq 0$ : (i) (32) holds if $D^{\prime \prime \prime}(e) \leq 0$ and $p_{e e e}(e, \theta) \geq 0$; and (ii) (33) holds if $D^{\prime \prime \prime}(e) \geq 0, p_{e e e}(e, \theta) \leq 0$, and $D^{\prime \prime \prime}(e)$ and $\left|p_{e e e}(e, \theta)\right|$ are sufficiently small.

Lemma 6. $\frac{d^{2} e_{1}}{d\left(\Delta_{1}\right)^{2}}<0$ and $\frac{d e_{12}}{d \Delta_{1}}>\frac{d e_{1}}{d \Delta_{1}}$ at the solution to [RP] when $\psi_{1}=1$ if for all $\theta$ and $e \geq 0$ : (i) $p_{e \theta}(e, \theta) \geq 0$; (ii) $p_{e e \theta}(e, \theta) \geq 0$; and (iii) $\left|D^{\prime \prime \prime}(e)\right|$ and $\left|p_{e e e}(e, \theta)\right|$ are sufficiently small.

Proof. The conclusion follows from Lemmas 4 and 5.

Lemma 7. Suppose the conditions in Lemma 6 hold. Then $h>0$ at the solution to [RP] when $\psi_{1}=1$, where

$$
\begin{aligned}
h \equiv \phi_{1} p_{e}\left(e_{1}, \theta_{1}\right) \frac{d e_{1}}{d \Delta_{1}} & -\left[\bar{c}-\underline{c}-\Delta_{1}\right] \phi_{1} \frac{d}{d \Delta_{1}}\left(p_{e}\left(e_{1}, \theta_{1}\right) \frac{d e_{1}}{d \Delta_{1}}\right) \\
& +\left[1-\phi_{1}\right]\left[p_{e}\left(e_{12}, \theta_{2}\right) \frac{d e_{12}}{d \Delta_{1}}-p_{e}\left(e_{1}, \theta_{1}\right) \frac{d e_{1}}{d \Delta_{1}}\right] .
\end{aligned}
$$

Proof. Conclusion 2 implies that $\bar{c}-\underline{c}>\Delta_{1}$ at the solution to $[\mathrm{RP}]$ when $\psi_{1}=1$. Therefore $h>0$ if:

$$
\frac{d}{d \Delta_{1}}\left(p_{e}\left(e_{1}, \theta_{1}\right) \frac{d e_{1}}{d \Delta_{1}}\right) \leq 0 \quad \text { and } \quad p_{e}\left(e_{12}, \theta_{2}\right) \frac{d e_{12}}{d \Delta_{1}} \geq p_{e}\left(e_{1}, \theta_{1}\right) \frac{d e_{1}}{d \Delta_{1}}
$$

Lemma 6 implies that $\frac{d^{2} e_{1}}{d\left(\Delta_{1}\right)^{2}}<0$ under the maintained conditions. Therefore:

$$
\frac{d}{d \Delta_{1}}\left(p_{e}\left(e_{1}, \theta_{1}\right) \frac{d e_{1}}{d \Delta_{1}}\right)=p_{e}\left(e_{1}, \theta_{1}\right) \frac{d^{2} e_{1}}{d\left(\Delta_{1}\right)^{2}}+p_{e e}\left(e_{1}, \theta_{1}\right)\left(\frac{d e_{1}}{d \Delta_{1}}\right)^{2}<0 .
$$

Lemma 6 implies that $\frac{d e_{12}}{d \Delta_{1}}>\frac{d e_{1}}{d \Delta_{1}}$ under the maintained conditions. Therefore, because $e_{12} \geq e_{1}$ when $p_{e \theta}(e, \theta) \geq 0$ from (8):

$$
p_{e}\left(e_{12}, \theta_{2}\right) \frac{d e_{12}}{d \Delta_{1}}=\frac{D^{\prime}\left(e_{12}\right)}{\Delta_{1}} \frac{d e_{12}}{d \Delta_{1}}>\frac{D^{\prime}\left(e_{1}\right)}{\Delta_{1}} \frac{d e_{1}}{d \Delta_{1}}=p_{e}\left(e_{1}, \theta_{1}\right) \frac{d e_{1}}{d \Delta_{1}} .
$$

(35), (36), and (37) imply that $h>0$ at the solution to [RP] under the maintained conditions. 
Proof of Conclusion 3. (19) implies that under the maintained conditions, the value of $\Delta_{1}$ at the solution to $[\mathrm{RP}]$ is determined by:

$$
\left[\bar{c}-\underline{c}-\Delta_{1}\right] \phi_{1} p_{e}\left(e_{1}, \theta_{1}\right) \frac{d e_{1}}{d \Delta_{1}}-\left[1-\phi_{1}\right]\left[p\left(e_{12}, \theta_{2}\right)-p\left(e_{1}, \theta_{1}\right)\right]=0 .
$$

From (8), $e_{1}$ and $e_{12}$ do not vary with $\phi_{1}$, given $\Delta_{1}$. Therefore, differentiating (38) provides:

$$
\left\{\left[\bar{c}-\underline{c}-\Delta_{1}\right] p_{e}\left(e_{1}, \theta_{1}\right) \frac{d e_{1}}{d \Delta_{1}}+p\left(e_{12}, \theta_{2}\right)-p\left(e_{1}, \theta_{1}\right)\right\} d \phi_{1}=h d \Delta_{1},
$$

where $h$ is defined in (34). $p\left(e_{12}, \theta_{2}\right)>p\left(e_{1}, \theta_{1}\right)$ when $p_{e \theta}(e, \theta) \geq 0$. Also, Conclusion 2 implies that $\bar{c}-\underline{c}>\Delta_{1}$ when $\psi_{1}=1$, and Lemma 7 implies that $h>0$. Therefore, (39) implies that $\frac{d \Delta_{1}}{d \phi_{1}}>0$.

Proof of Conclusion 4. Differentiating (38) provides:

$$
-\left[1-\phi_{1}\right]\left[p_{e}\left(e_{12}, \theta_{2}\right) \frac{d e_{12}}{d \theta_{2}}+p_{\theta}\left(e_{12}, \theta_{2}\right)\right] d \theta_{2}=h d \Delta_{1} \text {. }
$$

Lemma 7 implies that $h>0$. Furthermore, (8) implies that $\frac{d e_{12}}{d \theta_{2}} \geq 0$. Therefore, (40) implies that $\frac{d \Delta_{1}}{d \theta_{2}}<0$.

Differentiating (38) also provides:

$$
\begin{aligned}
\left\{\left[\bar{c}-\underline{c}-\Delta_{1}\right] \phi_{1} \frac{d}{d \theta_{1}}\left(p_{e}\left(e_{1}, \theta_{1}\right) \frac{d e_{1}}{d \Delta_{1}}\right)\right. & \left.+\phi_{2}\left[p_{e}\left(e_{1}, \theta_{1}\right) \frac{d e_{1}}{d \theta_{1}}+p_{\theta}\left(e_{1}, \theta_{1}\right)\right]\right\} d \theta_{1} \\
& =h d \Delta_{1} .
\end{aligned}
$$

$h>0$ under the maintained conditions. Furthermore, Conclusion 2 implies that $\bar{c}-\underline{c}>\Delta_{1}$ when $\psi_{1}=1$. In addition, (8) implies that $\frac{d e_{1}}{d \theta_{1}} \geq 0$. Therefore, (41) implies that $\frac{d \Delta_{1}}{d \theta_{2}}>0$ if $\phi_{1} \frac{d}{d \theta_{1}}\left(p_{e}\left(e_{1}, \theta_{1}\right) \frac{d e_{1}}{d \Delta_{1}}\right)$ is nonnegative or sufficiently close to 0 .

$$
\frac{d}{d \theta_{1}}\left(p_{e}\left(e_{1}, \theta_{1}\right) \frac{d e_{1}}{d \Delta_{1}}\right)=p_{e}(\cdot) \frac{d}{d \theta_{1}}\left(\frac{d e_{1}}{d \Delta_{1}}\right)+\frac{d e_{1}}{d \Delta_{1}}\left[p_{e e}(\cdot) \frac{d e_{1}}{d \Delta_{1}}+p_{e \theta}(\cdot)\right] .
$$

The second of the two terms to the right of the equality in (42) is nonnegative or sufficiently close to 0 if $p_{e e}(\cdot)$ is sufficiently close to 0 . Furthermore, from (8):

$$
\begin{aligned}
\frac{d}{d \theta_{1}}\left(\frac{d e_{1}}{d \Delta_{1}}\right)= & \frac{d}{d \theta_{1}}\left(\frac{p_{e}\left(e_{1}, \theta_{1}\right)}{\Delta_{1}\left[D^{\prime \prime}\left(e_{1}\right)-\Delta_{1} p_{e e}\left(e_{1}, \theta_{1}\right)\right]}\right) \\
\stackrel{s}{=} & {\left[D^{\prime \prime}\left(e_{1}\right)-\Delta_{1} p_{e e}\left(e_{1}, \theta_{1}\right)\right]\left[p_{e e}(\cdot) \frac{d e_{1}}{d \theta_{1}}+p_{e \theta}(\cdot)\right] } \\
& -p_{e}(\cdot)\left[D^{\prime \prime \prime}\left(e_{1}\right) \frac{d e_{1}}{d \theta_{1}}-\Delta_{1} p_{e e e}(\cdot) \frac{d e_{1}}{d \theta_{1}}-\Delta_{1} p_{e e \theta}(\cdot)\right] .
\end{aligned}
$$

This expression is nonnegative or sufficiently close to 0 under the maintained conditions. 
Therefore, $\phi_{1} \frac{d}{d \theta_{1}}\left(p_{e}\left(e_{1}, \theta_{1}\right) \frac{d e_{1}}{d \Delta_{1}}\right)$ is nonnegative or sufficiently close to 0 under the maintained conditions.

Together, these findings imply that under the maintained conditions, $\frac{d \Delta_{1}}{d\left(\theta_{2}-\theta_{1}\right)}<0$ when $\psi_{1}=1$ at the solution to $[\mathrm{RP}]$.

Proof of Conclusion 5. Because $\Delta_{1}=\underline{r}_{1}-\bar{r}_{1}+\bar{c}-\underline{c}$, (19) implies that the value of $\Delta_{1}$ at the solution to $[\mathrm{RP}]$ is determined by:

$$
\begin{aligned}
& Z \equiv-\left[\underline{r}_{1}-\bar{r}_{1}\right] \phi_{1} p_{e}\left(e_{1}, \theta_{1}\right) \frac{d e_{1}}{d\left(\underline{r}_{1}-\bar{r}_{1}\right)}-\phi_{2}\left[p\left(e_{12}, \theta_{2}\right)-p\left(e_{1}, \theta_{1}\right)\right]=0 \\
& \Rightarrow \frac{d\left(\underline{r}_{1}-\bar{r}_{1}\right)}{d(\bar{c}-\underline{c})}=-\frac{\frac{\partial Z}{\partial(\bar{c}-\underline{c})}}{\frac{\partial Z}{\partial\left(\underline{r}_{1}-\bar{r}_{1}\right)}} .
\end{aligned}
$$

Differentiating (43) provides:

$$
\begin{aligned}
\frac{\partial Z}{\partial\left(\underline{r}_{1}-\bar{r}_{1}\right)}= & -\phi_{1} p_{e}\left(e_{1}, \theta_{1}\right) \frac{d e_{1}}{d\left(\underline{r}_{1}-\bar{r}_{1}\right)}-\phi_{1}\left[\underline{r}_{1}-\bar{r}_{1}\right] \frac{d}{d\left(\underline{r}_{1}-\bar{r}_{1}\right)}\left(p_{e}\left(e_{1}, \theta_{1}\right) \frac{d e_{1}}{d\left(\underline{r}_{1}-\bar{r}_{1}\right)}\right) \\
& -\phi_{2}\left[p_{e}\left(e_{12}, \theta_{2}\right) \frac{d e_{12}}{d\left(\underline{r}_{1}-\bar{r}_{1}\right)}-p_{e}\left(e_{1}, \theta_{1}\right) \frac{d e_{1}}{d\left(\underline{r}_{1}-\bar{r}_{1}\right)}\right]=-h<0 .
\end{aligned}
$$

The last equality in (45) holds because $\frac{d e_{1}}{d\left(\underline{r}_{1}-\bar{r}_{1}\right)}=\frac{d e_{1}}{d \Delta_{1}}, \frac{d e_{12}}{d\left(\underline{r}_{1}-\bar{r}_{1}\right)}=\frac{d e_{12}}{d \Delta_{1}}$, and $\underline{r}_{1}-\bar{r}_{1}=$ $-\left(\bar{c}-\underline{c}-\Delta_{1}\right)$, since $\Delta_{1}=\underline{r}_{1}-\bar{r}_{1}+\bar{c}-\underline{c}$. The inequality in (45) reflects Lemma 7 .

Differentiating (43) also provides:

$$
\begin{aligned}
\frac{\partial Z}{\partial(\bar{c}-\underline{c})}= & \phi_{1}\left[\bar{r}_{1}-\underline{r}_{1}\right] \frac{d}{d(\bar{c}-\underline{c})}\left(p_{e}\left(e_{1}, \theta_{1}\right) \frac{d e_{1}}{d\left(\underline{r}_{1}-\bar{r}_{1}\right)}\right) \\
& -\phi_{2}\left[p_{e}\left(e_{12}, \theta_{2}\right) \frac{d e_{12}}{d(\bar{c}-\underline{c})}-p_{e}\left(e_{1}, \theta_{1}\right) \frac{d e_{1}}{d(\bar{c}-\underline{c})}\right]<0 .
\end{aligned}
$$

Conclusion 2 implies that $\bar{r}_{1}>\underline{r}_{1}$. Therefore, the inequality in (46) follows from (36) and (37) under the maintained conditions because $\frac{d e_{1}}{d(\bar{c}-\underline{c})}=\frac{d e_{1}}{d \Delta_{1}}, \frac{d e_{12}}{d(\bar{c}-\underline{c})}=\frac{d e_{12}}{d \Delta_{1}}$, and $\frac{d e_{1}}{d\left(\underline{r}_{1}-\bar{r}_{1}\right)}=\frac{d e_{1}}{d \Delta_{1}}$, since $\Delta_{1}=\underline{r}_{1}-\bar{r}_{1}+\bar{c}-\underline{c} . \quad(44)$, (45), and (46) imply that $\frac{d\left(\underline{r}_{1}-\bar{r}_{1}\right)}{d(\bar{c}-\underline{c})}<0$. Therefore, $\frac{d \Delta_{1}}{d(\bar{c}-\underline{c})}=\frac{d\left(\underline{r}_{1}-\bar{r}_{1}+\bar{c}-\underline{c}\right)}{d(\bar{c}-\underline{c})}<1$.

Proof of Conclusion 6. Differentiating (38) provides:

$$
-\phi_{2} p_{e}\left(e_{12}, \theta_{2}\right) \frac{d e_{12}}{d \delta_{2}} d \delta_{2}=h d \Delta_{1}
$$

Lemma 7 implies that $h>0$. Therefore, (47) implies that $\frac{d \Delta_{1}}{d \delta_{2}}>0$ when $\psi_{1}=1$ at the solution to $[\mathrm{RP}]$. 
Differentiating (38) also provides:

$$
\left\{\left[\bar{c}-\underline{c}-\Delta_{1}\right] \phi_{1} \frac{d}{d \delta_{1}}\left(p_{e}\left(e_{1}, \theta_{1}\right) \frac{d e_{1}}{d \Delta_{1}}\right)+\phi_{2} p_{e}\left(e_{1}, \theta_{1}\right) \frac{d e_{1}}{d \delta_{1}}\right\} d \delta_{1}=h d \Delta_{1} .
$$

$h>0$ and $\bar{c}-\underline{c}>\Delta_{1}$ (from Conclusion 2). Furthermore:

$$
\frac{d}{d \delta_{1}}\left(p_{e}\left(e_{1}, \theta_{1}\right) \frac{d e_{1}}{d \Delta_{1}}\right)=p_{e}\left(e_{1}, \theta_{1}\right) \frac{d}{d \delta_{1}}\left(\frac{d e_{1}}{d \Delta_{1}}\right)+\frac{d e_{1}}{d \Delta_{1}} p_{e e}\left(e_{1}, \theta_{1}\right) \frac{d e_{1}}{d \delta_{1}}
$$

From (8):

$$
\begin{aligned}
& \frac{d}{d \delta_{1}}\left(\frac{d e_{1}}{d \Delta_{1}}\right)=\frac{d}{d \delta_{1}}\left(\frac{p_{e}\left(e_{1}, \theta_{1}\right)}{\Delta_{1}\left[D^{\prime \prime}\left(e_{1}\right)-\Delta_{1} p_{e e}\left(e_{1}, \theta_{1}\right)\right]}\right) \\
& \stackrel{s}{=}\left[D^{\prime \prime}\left(e_{1}\right)-\Delta_{1} p_{e e}\left(e_{1}, \theta_{1}\right)\right] p_{e e}(\cdot) \frac{d e_{1}}{d \delta_{1}}-p_{e}(\cdot)\left[D^{\prime \prime \prime}\left(e_{1}\right) \frac{d e_{1}}{d \delta_{1}}-\Delta_{1} p_{e e e}(\cdot) \frac{d e_{1}}{d \delta_{1}}\right] .
\end{aligned}
$$

Because $\frac{d e_{1}}{d \delta_{1}}<0$, the expression in (50) is positive when the conditions in Lemma 6 hold. Therefore, (49) implies that $\frac{d}{d \delta_{1}}\left(p_{e}\left(e_{1}, \theta_{1}\right) \frac{d e_{1}}{d \Delta_{1}}\right)>0$ under these conditions. Consequently, (48) implies that: (i) $\frac{d \Delta_{1}}{d \delta_{1}}>0$ when $\phi_{1}$ is sufficiently large; and (ii) $\frac{d \Delta_{1}}{d \delta_{1}}<0$ when $\phi_{2}$ is sufficiently large.

These findings imply that $\frac{d \Delta_{1}}{d \delta}>0$ when $\phi_{1}$ is sufficiently large.

Proof of Conclusion 7. Differentiating (38) provides:

$$
\left\{\left[\bar{c}-\underline{c}-\Delta_{1}\right] \phi_{1} \frac{d}{d \alpha}\left(\alpha \frac{d e_{1}}{d \Delta_{1}}\right)-\phi_{2} \alpha\left[\frac{d e_{12}}{d \alpha}-\frac{d e_{1}}{d \alpha}\right]\right\} d \alpha=h d \Delta_{1},
$$

where $h>0$, from Lemma 7. From (8):

$$
\alpha \Delta_{1}=D^{\prime}\left(e_{1}\right)=D^{\prime}\left(e_{12}\right) \Rightarrow \frac{d e_{1}}{d \alpha}=\frac{d e_{12}}{d \alpha}=\frac{\Delta_{1}}{D^{\prime \prime}\left(e_{1}\right)}=\frac{\Delta_{1}}{D^{\prime \prime}\left(e_{12}\right)}>0 .
$$

Because $\bar{c}-\underline{c}>\Delta_{1}$ from Conclusion 2, (51) and (52) imply:

$$
\frac{d \Delta_{1}}{d \alpha} \stackrel{s}{=} \frac{d}{d \alpha}\left(\alpha \frac{d e_{1}}{d \Delta_{1}}\right)=\alpha \frac{d}{d \alpha}\left(\frac{d e_{1}}{d \Delta_{1}}\right)+\frac{d e_{1}}{d \Delta_{1}}
$$

From (8):

$$
\frac{d}{d \alpha}\left(\frac{d e_{1}}{d \Delta_{1}}\right)=\frac{d}{d \alpha}\left(\frac{\alpha}{\Delta_{1} D^{\prime \prime}\left(e_{1}\right)}\right) \stackrel{s}{=} D^{\prime \prime}\left(e_{1}\right)-\alpha D^{\prime \prime \prime}\left(e_{1}\right) \frac{d e_{1}}{d \alpha}>0 .
$$

The inequality in (54) holds because $D^{\prime \prime \prime}\left(e_{1}\right)$ is sufficiently small, by assumption. (52), (53), and (54) imply $\frac{d \Delta_{1}}{d \alpha}>0$. 
Proof of Conclusion 9. The regulator's problem in this setting, [RP1], is:

$$
\underset{\underline{r}, \bar{r}}{\operatorname{Maximize}}-\left[\phi_{1} p\left(e_{1}, \theta_{1}\right)+\phi_{2} p\left(e_{2}, \theta_{2}\right)\right] \underline{r}-\left[1-\phi_{1} p\left(e_{1}, \theta_{1}\right)-\phi_{2} p\left(e_{2}, \theta_{2}\right)\right] \bar{r}
$$

subject to, for $i \in\{1,2\}$ :

$$
p\left(e_{i}, \theta_{i}\right)[\underline{r}-\underline{c}]+\left[1-p\left(e_{i}, \theta_{i}\right)\right][\bar{r}-\bar{c}]-D\left(e_{i}\right) \geq 0 .
$$

Let $\lambda_{i}$ denote the Lagrange multiplier associated with constraint (55). Also let [RP1] denote problem [RP1] with constraint (55) omitted for $i=2$. The necessary conditions for a solution to $[\mathrm{RP} 1]^{\prime}$ include:

$$
\begin{gathered}
\underline{r}: \quad-\left[\phi_{1} p\left(e_{1}, \theta_{1}\right)+\phi_{2} p\left(e_{2}, \theta_{2}\right)\right]+\phi_{1} p_{e}\left(e_{1}, \theta_{1}\right)[\bar{r}-\underline{r}] \frac{d e_{1}}{d \underline{r}} \\
+\phi_{2} p_{e}\left(e_{2}, \theta_{2}\right)[\bar{r}-\underline{r}] \frac{d e_{2}}{d \underline{r}}+\lambda_{1} p\left(e_{1}, \theta_{1}\right)=0 ; \\
\bar{r}: \quad-\left[1-\phi_{1} p\left(e_{1}, \theta_{1}\right)-\phi_{2} p\left(e_{2}, \theta_{2}\right)\right]+\phi_{1} p_{e}\left(e_{1}, \theta_{1}\right)[\bar{r}-\underline{r}] \frac{d e_{1}}{d \bar{r}} \\
+\phi_{2} p_{e}\left(e_{2}, \theta_{2}\right)[\bar{r}-\underline{r}] \frac{d e_{2}}{d \bar{r}}+\lambda_{1}\left[1-p\left(e_{1}, \theta_{1}\right)\right]=0 .
\end{gathered}
$$

Summing (56) and (57), using (8), provides $\lambda_{1}=1$. Therefore, (56) can be written as:

$$
[\bar{r}-\underline{r}]\left[\phi_{1} p_{e}\left(e_{1}, \theta_{1}\right) \frac{d e_{1}}{d \underline{r}}+\phi_{2} p_{e}\left(e_{2}, \theta_{2}\right) \frac{d e_{2}}{d \underline{r}}\right]=\phi_{2}\left[p\left(e_{2}, \theta_{2}\right)-p\left(e_{1}, \theta_{1}\right)\right] .
$$

Furthermore, from (55):

$$
p\left(e_{1}, \theta_{1}\right)[\underline{r}-\underline{c}]+\left[1-p\left(e_{1}, \theta_{1}\right)\right][\bar{r}-\bar{c}]-D\left(e_{1}\right)=0 .
$$

(8) implies that $p\left(e_{2}, \theta_{2}\right)>p\left(e_{1}, \theta_{1}\right)$. Therefore, (8) and (58) imply that $\bar{r}>\underline{r}$ at the solution to $[\mathrm{RP} 1]^{\prime}$.

Finally, to verify that the solution to [RP1]' is the solution to [RP1], it suffices to prove that $p\left(e_{2}, \theta_{2}\right)[\underline{r}-\underline{c}]+\left[1-p\left(e_{2}, \theta_{2}\right)\right][\bar{r}-\bar{c}]-D\left(e_{2}\right) \geq 0$ at the solution to [RP1]'.

If $\underline{r}-\underline{c}=\bar{r}-\bar{c}$ at the solution to [RP1]', then $e_{1}=e_{2}=0$. Consequently, from (59):

$$
\begin{aligned}
p\left(e_{2}, \theta_{2}\right)[\underline{r}-\underline{c}]+\left[1-p\left(e_{2}, \theta_{2}\right)\right][\bar{r}-\bar{c}]-D\left(e_{2}\right) \\
=\underline{r}-\underline{c}=p\left(e_{1}, \theta_{1}\right)[\underline{r}-\underline{c}]+\left[1-p\left(e_{1}, \theta_{1}\right)\right][\bar{r}-\bar{c}]-D\left(e_{1}\right)=0 .
\end{aligned}
$$

If $\underline{r}-\underline{c}>\bar{r}-\bar{c}$ at the solution to [RP1]', then the maintained assumptions ensure $\max \{p(e, \theta) \underline{R}+[1-p(e, \theta)] \bar{R}-D(e)\}$ is strictly increasing in $\theta$ for all $\underline{R}>\bar{R}$. Consequently:

$$
p\left(e_{2}, \theta_{2}\right)[\underline{r}-\underline{c}]+\left[1-p\left(e_{2}, \theta_{2}\right)\right][\bar{r}-\bar{c}]-D\left(e_{2}\right)
$$




$$
>p\left(e_{1}, \theta_{1}\right)[\underline{r}-\underline{c}]+\left[1-p\left(e_{1}, \theta_{1}\right)\right][\bar{r}-\bar{c}]-D\left(e_{1}\right)=0 .
$$

Proof of Conclusion 10. Let $e^{0}$ denote the utility's effort supply at the solution to [RP1]. From (19) and (58), the values of $\Delta_{1}^{*}$ and $\Delta^{*}$ are determined, respectively, by:

$$
\begin{aligned}
& {\left[\bar{r}_{1}-\underline{r}_{1}\right] \phi_{1} p_{e}\left(e_{1}, \theta_{1}\right) \frac{d e_{1}}{d \underline{r}_{1}}=\phi_{2}\left[p\left(e_{12}, \theta_{2}\right)-p\left(e_{1}, \theta_{1}\right)\right], \text { and }} \\
& {[\bar{r}-\underline{r}]\left[\phi_{1} p_{e}\left(e_{1}^{0}, \theta_{1}\right) \frac{d e_{1}^{0}}{d \underline{r}}+\phi_{2} p_{e}\left(e_{2}^{0}, \theta_{2}\right) \frac{d e_{2}^{0}}{d \underline{r}}\right]=\phi_{2}\left[p\left(e_{2}^{0}, \theta_{2}\right)-p\left(e_{1}^{0}, \theta_{1}\right)\right] .}
\end{aligned}
$$

Suppose $\Delta^{*}=\Delta_{1}^{*}$. Then $\bar{r}_{1}-\underline{r}_{1}=\bar{r}-\underline{r}$. Furthermore, $e_{1}=e_{1}^{0}, e_{12}=e_{2}^{0}$, and $\frac{d e_{1}}{d \underline{r}_{1}}=\frac{d e_{1}^{0}}{d \underline{r}}$, from (8). Therefore, (60) and (61) imply:

$$
[\bar{r}-\underline{r}] \phi_{2} p_{e}\left(e_{2}^{0}, \theta_{2}\right) \frac{d e_{2}^{0}}{d \underline{r}}=0 \Rightarrow \bar{r}=\underline{r} .
$$

From (61), this conclusion implies $p\left(e_{2}^{0}, \theta_{2}\right)=p\left(e_{1}^{0}, \theta_{1}\right)$, which violates the maintained assumptions. This contradiction implies $\Delta^{*} \neq \Delta_{1}^{*}$.

Now suppose $\Delta^{*}<\Delta_{1}^{*}$. Then because (8) implies $p\left(e_{12}, \theta_{2}\right)>p\left(e_{1}, \theta_{1}\right)$, (60) implies that at the solutions to $[\mathrm{RP}]$ and $[\mathrm{RP} 1]$ :

$$
\underline{r}-\underline{c}-(\bar{r}-\bar{c})<\underline{r}_{1}-\underline{c}-\left(\bar{r}_{1}-\bar{c}\right) \quad \Leftrightarrow \quad \bar{r}-\underline{r}>\bar{r}_{1}-\underline{r}_{1}>0 .
$$

In addition, $e_{1}>e_{1}^{0}$ from (8). Furthermore:

(63) holds because:

$$
p\left(e_{12}, \theta_{2}\right)-p\left(e_{1}, \theta_{1}\right)>p\left(e_{2}^{0}, \theta_{2}\right)-p\left(e_{1}^{0}, \theta_{1}\right) .
$$

$$
\begin{aligned}
\frac{d}{d \Delta_{1}}\left\{p\left(e_{12}, \theta_{2}\right)-p\left(e_{1}, \theta_{1}\right)\right\} & =p_{e}\left(e_{12}, \theta_{2}\right) \frac{d e_{12}}{d \Delta_{1}}-p_{e}\left(e_{1}, \theta_{1}\right) \frac{d e_{1}}{d \Delta_{1}} \\
& =\frac{D^{\prime}\left(e_{12}\right)}{\Delta_{1}}\left[\frac{d e_{12}}{d \Delta_{1}}-\frac{D^{\prime}\left(e_{1}\right)}{D^{\prime}\left(e_{12}\right)} \frac{d e_{1}}{d \Delta_{1}}\right]>0 .
\end{aligned}
$$

This inequality holds because $\frac{d e_{12}}{d \Delta_{1}}>\frac{d e_{1}}{d \Delta_{1}}$ from Lemma 6 and because $D^{\prime}\left(e_{12}\right)>D^{\prime}\left(e_{1}\right)$ since $e_{12}>e_{1}$, from (8).

(60), (61), (62), and (63) imply:

$$
\phi_{1} p_{e}\left(e_{1}, \theta_{1}\right) \frac{d e_{1}}{d \underline{r}_{1}}>\phi_{1} p_{e}\left(e_{1}^{0}, \theta_{1}\right) \frac{d e_{1}^{0}}{d \underline{r}}+\phi_{2} p_{e}\left(e_{2}^{0}, \theta_{2}\right) \frac{d e_{2}^{0}}{d \underline{r}} .
$$

This inequality cannot hold because $\phi_{2} p_{e}\left(e_{2}^{0}, \theta_{2}\right) \frac{d e_{2}^{0}}{d \underline{r}}>0$ and:

$$
p_{e}\left(e_{1}^{0}, \theta_{1}\right) \frac{d e_{1}^{0}}{d \underline{r}} \geq p_{e}\left(e_{1}, \theta_{1}\right) \frac{d e_{1}}{d \underline{r}_{1}} .
$$

This inequality holds because when $e_{1}>e_{1}^{0}$ : (i) $p_{e}\left(e_{1}^{0}, \theta_{1}\right) \geq p_{e}\left(e_{1}, \theta_{1}\right)$ since $p_{e e}\left(e, \theta_{1}\right) \leq 0$; and (ii) $\frac{d e_{1}^{0}}{d \underline{r}} \geq \frac{d e_{1}}{d \underline{r}_{1}}$ since $\frac{d^{2} e_{1}}{d\left(\Delta_{1}\right)^{2}} \leq 0$ from Lemma 6 . 
Therefore, by contradiction, $\Delta^{*}>\Delta_{1}^{*}$.

Proof of Conclusion 11. We will show that the identified compensation structure satisfies all of the necessary conditions for a solution to [RP] in the present setting. Constraint (11) is satisfied for $i=1,2$ because the compensation structure does not vary with the utility's report of $\theta$.

Because $p\left(e, \theta_{2}\right)>p\left(e, \theta_{1}\right)$ for all $e \geq 0$ :

$$
p\left(e_{1}^{*}, \theta_{1}\right)=p\left(e_{2}^{*}, \theta_{2}\right) \Rightarrow e_{1}^{*}>e_{2}^{*} \Rightarrow D\left(e_{1}^{*}\right)>D\left(e_{2}^{*}\right) .
$$

Therefore, letting $r$ denote the fixed payment under the identified compensation structure:

$$
r-\bar{c}+p\left(e_{2}^{*}, \theta_{2}\right)[\bar{c}-\underline{c}]-D\left(e_{2}^{*}\right)>r-\bar{c}+p\left(e_{1}^{*}, \theta_{1}\right)[\bar{c}-\underline{c}]-D\left(e_{1}^{*}\right)=0 .
$$

(64) implies that (10) is satisfied for $i=1,2$.

Let $\lambda_{i}$ and $\lambda_{i j}$ denote the Lagrange multipliers associated with constraints (10) and (11), respectively. It is readily verified that the necessary conditions for a solution to [RP] with respect to $\underline{r}_{i}$ and $\bar{r}_{i}$ are, for $i, j \in\{1,2\}(j \neq i)$ :

$$
\begin{aligned}
& {\left[-\phi_{i}+\lambda_{i}+\lambda_{i j}\right] p\left(e_{i}, \theta_{i}\right)-\lambda_{j i} p\left(e_{i j}, \theta_{j}\right)+\phi_{i} p_{e}\left(e_{i}, \theta_{i}\right)\left[\bar{r}_{i}-\underline{r}_{i}\right] \frac{d e_{i}}{d \underline{r}_{i}}=0 ;} \\
& {\left[-\phi_{i}+\lambda_{i}+\lambda_{i j}\right]\left[1-p\left(e_{i}, \theta_{i}\right)\right]-\lambda_{j i}\left[1-p\left(e_{i j}, \theta_{j}\right)\right]+\phi_{i} p_{e}\left(e_{i}, \theta_{i}\right)\left[\bar{r}_{i}-\underline{r}_{i}\right] \frac{d e_{i}}{d \bar{r}_{i}}=0 .}
\end{aligned}
$$

Adding (65) and (66) provides, for $i, j \in\{1,2\}(j \neq i)$ :

$$
\lambda_{i}+\lambda_{i j}=\phi_{i}+\lambda_{j i} \Rightarrow \lambda_{1}+\lambda_{2}=1 .
$$

(65) and (67) imply that for $i, j \in\{1,2\}(j \neq i)$ :

$$
\lambda_{j i}\left[p\left(e_{i j}, \theta_{j}\right)-p\left(e_{i}, \theta_{i}\right)\right]=\phi_{i} p_{e}\left(e_{i}, \theta_{i}\right)\left[\bar{r}_{i}-\underline{r}_{i}\right] \frac{d e_{i}}{d \bar{r}_{i}} .
$$

(64) implies that $\lambda_{2}=0$, so $\lambda_{1}=1$ from (67).

Finally, observe that (68) is satisfied for any $\lambda_{12} \geq 0$ and $\lambda_{21} \geq 0$ because with $\Delta_{1}=$ $\Delta_{2}=\bar{c}-\underline{c}:$

$$
\begin{aligned}
& p\left(e_{12}, \theta_{2}\right)-p\left(e_{1}, \theta_{1}\right)=p\left(e_{2}^{*}, \theta_{2}\right)-p\left(e_{1}^{*}, \theta_{1}\right)=0, \quad \text { and } \\
& p\left(e_{21}, \theta_{1}\right)-p\left(e_{2}, \theta_{2}\right)=p\left(e_{1}^{*}, \theta_{1}\right)-p\left(e_{2}^{*}, \theta_{2}\right)=0 .
\end{aligned}
$$

Proof of Conclusion 12. The proof is by example. We view $[R P]$ as a nonlinear mixed complementary program (MCP) and employ the GAMS software and the PATH algorithm (Ferris and Munson, 2000) to solve the MCP. Because the solutions to nonlinear MCPs can be sensitive to the initial values of the endogenous variables used in the PATH algorithm, we employ a Monte Carlo algorithm to randomly select 10,000 initial values for all endogenous variables. We solve the nonlinear MCP for each set of initial values and select the solution with the lowest expected procurement cost. 
We consider a setting where $p\left(e, \theta_{i}\right)=0.25 \theta+0.25 e-\gamma e \theta, D(e)=4,500 e^{2}, \theta_{1}=$ 0.25 , and $\theta_{2}=\phi_{1}=\phi_{2}=0.5$. The values of $\underline{r}_{1}, \bar{r}_{1}, \underline{r}_{2}$, and $\bar{r}_{2}$ at the solution to [RP] in this setting are presented in Table A1 for selected values of $\gamma$.

\begin{tabular}{|l|c|l|l|l|l|}
\hline & $\gamma=0$ & $\gamma=0.4$ & $\gamma=\widehat{\gamma}$ & $\gamma=0.5$ & $\gamma=0.075$ \\
\hline$\underline{r}_{1}$ & 86,559 & 91,220 & 92,316 & 92,509 & 95,984 \\
\hline $\bar{r}_{1}$ & 95,559 & 92,969 & 92,316 & 92,200 & 90,015 \\
\hline$\underline{r}_{2}$ & 88,465 & 91,731 & 92,316 & 92,413 & 93,846 \\
\hline $\bar{r}_{2}$ & 88,465 & 91,731 & 92,316 & 92,413 & 93,846 \\
\hline
\end{tabular}

Table A1. The Solution to $[\mathrm{RP}]$ for the Identified Value of $\gamma$.

$\widehat{\gamma} \approx 0.048533205$ in Table A1 is the value of $\gamma$ at which $p\left(e_{1}^{*}, \theta_{1}\right)=p\left(e_{2}^{*}, \theta_{2}\right)$ in this setting. As per Conclusion 11, $\bar{r}_{1}=\underline{r}_{1}=\bar{r}_{2}=\underline{r}_{2}$ when $\gamma=\widehat{\gamma}$. When the extent to which $e$ and $\theta$ are substitutes is sufficiently limited (i.e., when $\gamma<\widehat{\gamma}$ ), $\underline{r}_{1}<\bar{r}_{1}$. In contrast, $\underline{r}_{1}>\bar{r}_{1}$ for the indicated values of $\gamma$ above $\widehat{\gamma}$. 


\section{References}

Arizona Corporation Commission, In the Matter of Arizona Public Service Company for Approval of its 2014 Renewable Energy Standard Implementation Plan for RESET of Renewable Energy Adjustor. Docket No. E-01345A-12-0140, December 23, 2014.

Bower, Anthony, "Procurement Policy and Contracting Efficiency," International Economic Review, 34(4), November 1993, 873-901.

Brown, David and David Sappington, "Designing Compensation for Distributed Solar Generation: Is Net Metering Ever Optimal?" The Energy Journal, 38(3), May 2017a, 1-32.

Brown, David and David Sappington, "Technical Appendix to Accompany 'Optimal Procurement of Distributed Energy Resources'," 2017b (https://uofa.ualberta.ca/arts/about/peoplecollection/david-brown).

California Public Utilities Commission, Distribution Resources Plan R.14-08-013, 2013 (http: //www.cpuc.ca.gov/General.aspx?id=5071).

California Public Utilities Commission, Assigned Commissioner's Ruling Introducing a Draft Regulatory Incentives Proposal Discussion and Comment. Rulemaking 14-10-003, April 2016a.

California Public Utilities Commission, Decision Addressing Competitive Solicitation Framework and Utility Regulatory Incentive Pilot. Rulemaking 14-10-003, December 2016b.

Chu, Leon Yang and David Sappington, "Simple Cost-Sharing Contracts," American Economic Review, 97(1), March 2007, 419-428.

Commonwealth of Massachusetts, An Act Relative to Local Energy Investment and Infrastructure Modernization, Bill H.175 in the 190th Court, Introduced January 23, 2017.

Cossent, Rafael and Tomas Gomez, "Implementing Incentive Compatible Menus of Contracts to Regulate Electricity Distribution Investments," Utilities Policy, 27, December 2013, 28-38.

Costa, Paulo, Nuno Bento, and Vitor Marques, "The Impact of Regulation on a Firm's Incentive to Invest in Emergent Smart Grid Technologies," The Energy Journal, 38(2), 2017, 149-174.

Couture, Toby and Yves Gagnon, "An Analysis of Feed-in Tariff Remuneration Models: Implications for Renewable Energy Investment," Energy Policy, 38(3), February 2010, 955965.

Crouch, Martin, "Investment under RPI-X: Practical Experience with an Incentive Compatible Approach in the GB Electricity Distribution Sector," Utilities Policy, 14(4), December 2006, 240-244. 
Ferris, Michael and Todd Munson, "Complementarity Problems in GAMS and the PATH Solver," Journal of Economic Dynamics and Control, 24(2), February 2000, 165-188.

Hawaii Public Utilities Commission, Instituting a Proceeding to Investigate Distributed Energy Resource Policies, Docket No. 2014-0192, August 24, 2014 (http://dms.puc.hawaii.gov/ $\mathrm{dms} /$ DocketSearch?V_DocketNumber $=2014-0192 \& V \_D o c k e t T y p e=A l l \& V \_I n d u s t r y T y p e$ $=$ All\&V_Status=All\&AllWordsGroup=0\&QuickLink=1).

Jenkins, Jesse and Ignacio Perez-Arriaga, "Improved Regulatory Approaches for the Remuneration of Electricity Distribution Utilities with High Penetrations of Distributed Energy Resources," The Energy Journal, 38(3), May 2017, 63-91.

Joskow, Paul, "Incentive Regulation and Its Application to Electricity Networks," Review of Network Economics, 7(4), December 2008, 547-560.

Laffont, Jean-Jacques and David Martimort, The Theory of Incentives: The Principal-Agent Model. Princeton, NJ: Princeton University Press, 2002.

Laffont, Jean-Jacques and Jean Tirole, A Theory of Incentives in Procurement and Regulation. Cambridge, MA: MIT Press, 1993.

Lowry, Mark and Tim Woolf, "Performance-Based Regulation in a High Distributed Energy Resources Future," Lawrence Berkeley National Laboratory Report \#1004130, January 2016.

MIT Energy Initiative, Utility of the Future: An MIT Energy Initiative Response to an Industry in Transition. Cambridge, MA, 2016.

Myerson, Roger, "Incentive Compatibility and the Bargaining Problem," Econometrica, 47(1), February 1979, 61-73.

New York Public Service Commission, Developing the REV Market in New York: DPS Staff Straw Proposal on Track One Issues, Case 14-M-0101 - Proceeding on Motion of the Commission in Regard to Reforming the Energy Vision, August 22, $2014 \mathrm{a}$.

New York Public Service Commission, "Petition of Consolidated Edison Company of New York, Inc. for Approval of Brooklyn Queens Demand Management Program," Order Establishing Brooklyn/Queens Demand Management Program, Case 14-E-0302, December 12, 2014b.

New York Public Service Commission, Order Adopting Regulatory Policy Framework and Implementation Plan, Case 14-M-0101 - Proceeding on Motion of the Commission in Regard to Reforming the Energy Vision, February 26, 2015.

New York Public Service Commission, Order Adopting Ratemaking and Utility Revenue Model Policy Framework, Case 14-M-0101 - Proceeding on Motion of the Commission in Regard to Reforming the Energy Vision, Issued May 19, 2016. 
Ofgem, Electricity Distribution Price Control Review: Final Proposals - Incentives and Obligations, Decision Document 145/09, December 7, 2009.

Poullikkas, Andreas, "A Comparative Assessment of Net Metering and Feed in Tariff Schemes for Residential PV Systems," Sustainable Energy Technologies and Assessments, 3, September 2013, 1-8.

Reichelstein, Stefan, "Constructing Incentive Schemes for Government Contracts: An Application of Agency Theory," Accounting Review, 67(4), October 1992, 712-31.

Rogerson, William, "Simple Menus of Contracts in Cost-Based Procurement and Regulation," American Economic Review, 93(3), June 2003, 919-926.

Ruester, Sophia, Ignacio Perez-Arriaga, Sebastian Schwenen, Carlos Batlle, and Jean-Michel Glachant, "From Distribution Networks to Smart Distribution Systems: Rethinking the Regulation of European Electricity DSOs," Utilities Policy, 31, December 2014, 229-237.

Sappington, David, "Optimal Regulation of Research and Development Under Imperfect Information," Bell Journal of Economics, 13(2), Autumn 1982, 354-368.

Sappington, David and Dennis Weisman, Designing Incentive Regulation for the Telecommunications Industry. Cambridge, MA: MIT Press, 1996.

Yamamoto, Yoshihiro, "Pricing Electricity from Residential Photovoltaic Systems: A Comparison of Feed-in Tariffs, Net Metering, and Net Purchase and Sale," Solar Energy, 86(9), September 2012, 2678-2685. 


\section{Department of Economics, University of Alberta Working Paper Series}

2017-04: The Impact of Schooling Intensity on Student Learning: Evidence from a QuasiExperiment - Andrietti, V., Su, $\mathbf{X}$.

2017-03: The Voting Rights of Ex-Felons and Election Outcomes in the United States Klumpp, T., Mialon, H., Williams, $M$.

2017-02: Does the Design of a Fiscal Rule Matter for Welfare? - Landon, S., Smith, C.

2017-01: Carbon Pricing with an Output Subsidy under Imperfect Competition: The Case of Alberta's Restructured Electricity Market - Brown, D., Eckert, A., Eckert, H.

2016-18: Monetary Policy Tradeoffs Between Financial Stability and Price Stability Shukayev, M., Ueberfeldt, A.

2016-17: Managing Risk Taking with Interest Rate Policy and Macroprudential Regulations Cociuba, S., Shukayev, M., Ueberfeldt, A.

2016-16: On the Role of Maximum Demand Charges in the Presence of Distributed Generation Resources - Brown, D., Sappington, D.

2016-15: Implementing Cross-Border Interbank Lending in BoC-GEM-FIN - Shukayev, M., Toktamyssov, A.

2016-14: The Effects of Early Pregnancy on Education, Physical Health and Mental Distress: Evidence from Mexico - Gunes, P., Tsaneva, M.

2016-13: An Equilibrium Selection Theory of Monopolization - Eckert, A., Klumpp, T., Su, $\mathrm{X}$.

2016-12: Education Curriculum and Student Achievement: Theory and Evidence - Andrietti, V., Su, $\mathbf{X}$.

2016-11: Poverty and Aging - Marchand, J., Smeeding, T.

2016-10: Local Labor Markets and Natural Resources: A Synthesis of the Literature Marchand, J., Weber, J.

2016-09: Accounting for Firm Exit and Loss of Variety in the Welfare Cost of Regulations Andersen, D.

2016-08: Analyzing the I mpact of Electricity Market Structure Changes and Mergers: The Importance of Forward Commitments - Brown, D., Eckert, A.

2016-07: Credibility of History-Dependent Monetary Policies and Macroeconomic Instability Cateau, G., Shukayev, M.

2016-06: Electricity Market Mergers with Endogenous Forward Contracting - Brown, D., Eckert, A.

2016-05: Thinking about Minimum Wage Increases in Alberta: Theoretically, Empirically, and Regionally - Marchand, J.

2016-04: Economic and Socio-Demographic Determinants of Child Nutritional Status in Egypt: A Comprehensive Analysis using Quantile Regression Approach- Sharaf,M., Rashad,A.

2016-03: Regional Inequalities in Child Malnutrition in Egypt, J ordan, and Yemen: A BlinderOaxaca Decomposition Analysis - Rashad, A., Sharaf, M.

2016-02: Collateralized Borrowing and Risk Taking at Low Interest Rates - Cociuba, S., Shukayev, M., Ueberfeldt, A.

2016-01: Optimal Policies to Promote Efficient Distributed Generation of Electricity - Brown, D., Sappington, D.

2015-18: Departure and Promotion of U.S. Patent Examiners: Do Patent Characteristics Matter? - Langinier, C., Lluis, S.

2015-17: Socioeconomic Inequalities in Infant Mortality in Egypt: Analyzing Trends between 1995 and 2014 - Sharaf, M., Rashad, A.

2015-16: Does Economic Growth Reduce Child Malnutrition in Egypt? New Evidence from National Demographic and Health Survey - Rashad, A., Sharaf, M. 